\title{
Evolution of indoor cooking emissions captured by using secondary electrospray ionization high resolution mass spectrometry
}

Jiafa Zeng ${ }^{1}$, Zhujun $\mathrm{Yu}^{1}$, Majda Mekic ${ }^{2,3}$, Jiangping $\mathrm{Liu}^{2,3}$, Sheng $\mathrm{Li}^{2,3}$, Gwendal Loisel${ }^{2}$, Wei Gao ${ }^{1}$, Adrien Gandolfo $^{4}$, Zhen Zhou ${ }^{1}$, Xinming Wang ${ }^{2}$, Hartmut Herrmann ${ }^{5,6,7}$, Sasho Gligorovski ${ }^{2 *}$, Xue Li $^{1 *}$

${ }^{1}$ Institute of Mass Spectrometry and Atmospheric Environment; Guangdong Provincial Engineering Research Center for On-line Source Apportionment System of Air Pollution, Jinan University, Guangzhou 510632, China

${ }^{2}$ State Key Laboratory of Organic Geochemistry, Guangzhou Institute of Geochemistry, Chinese Academy of Sciences, Guangzhou 510640, China

${ }^{3}$ University of Chinese Academy of Sciences, Beijing 10069, China

${ }^{4}$ Aix Marseille Univ, CNRS, LCE, UMR 7376, 13331, Marseille, France

${ }^{5}$ School of Environmental Science and Engineering, Shandong University, Qingdao, 266237, China

${ }^{6}$ Shanghai Key Laboratory of Atmospheric Particle Pollution and Prevention, Department of

Environmental Science \& Engineering, Institute of Atmospheric Sciences, Fudan University, Shanghai 200433, China

${ }^{7}$ Leibniz-Institute for Tropospheric Research (TROPOS), Atmospheric Chemistry Department (ACD), Permoserstr. 15, 04318 Leipzig, Germany

The first three authors contribute equally

*Corresponding authors

E-mail: tamylee@jnu.edu.cn; gligorovski@gig.ac.cn

Keywords: Indoors, cooking emissions, $\mathrm{OH}$ radical, heterogeneous reactions, real-time monitoring 
2 Text S1: Detailed information about the cooking experiments and data analysis.

3 Table S1: Detection limit, sensitivity of SESI-HRMS, and sampling efficiency of 6.7-m

4 sampling tube.

5 Table S2. Detailed information of ions classified in Group 1 in Figure 1.

6 Table S3. Detailed information of ions classified in Group 2 in Figure 1.

$7 \quad$ Table S4. Detailed information of ions classified in Group 3 in Figure 1.

$8 \quad$ Table S5. Detailed information of ions classified in Group 4 in Figure 1.

9 Table S6. Detailed information of ions classified in Group 5 in Figure 1.

10 Table S7. Detailed information of ions classified in Group 6 in Figure 1.

11 Table S8. Detailed information of enhancement ratios (ER) of identified compounds.

12 Figure S1. Experiment setup of the cooking experiments.

13 Figure S2. Time-signal intensity profiles of (a) unsaturated aldehydes, (b) unsaturated fatty acids, (c) saturated fatty acids and (d) N-containing heterocyclic compounds detected during peanut oil cooking, which are consistent with those reported in previous studies

Figure S3. Mass spectral fingerprints of the peanut oil cooking experiment.

Figure S4. Time-signal intensity profiles of saturated and unsaturated fatty acids.

Figure S5. Cluster plot of ions detected in the control experiment.

Figure S6. Time-signal intensity profiles of ions detected at $m / z \quad 60.0816$ and $m / z, 133.1335$.

Figure S7. KMD plot of even $\mathrm{m} / \mathrm{z}$ values of ions detected under negative ion detection mode.

Figure S8. KMD plot of even $\mathrm{m} / \mathrm{z}$ values of ions detected under positive ion detection mode.

Figure S9. KMD plot of odd $\mathrm{m} / \mathrm{z}$ values of ions detected under positive ion detection mode.

\begin{tabular}{|l|l|}
\hline Number of Pages & 29 \\
\hline Number of Text & 1 \\
\hline Number of Tables & 8 \\
\hline Number of Figures & 9 \\
\hline
\end{tabular}


Txet S1: Detailed information about the cooking experiments and data analysis.

Experiment setup. Cooking experiment were conducted in a $23 \mathrm{~m} 3$ room with a window $(0.99 \mathrm{~m} 2)$ face to south-east at our institute (Figure S1). The sunlight irradiated the room through the window during certain periods of the day. Peanut oil from a local supermarket was heated in a pan on a natural gas stove (ZB-16M, Iwatani, Japan) for about $10 \mathrm{~min}$, and then the gas stove was turned off. Two fans were placed at two corners of the room (Figure S1) to ensure the indoor air was homogeneously mixed during the experiments.

Carbon dioxide $\left(\mathrm{CO}_{2}\right)$ emitted during the combustion process was recorded by a single photon ionization time-of-flight mass spectrometer (SPIMS 3000, Hexin Analytical Instrument Co., Ltd., China) at a time resolution of $1 \mathrm{~s}$ to calculate the air-exchange rate in the room. Since the outside $\mathrm{CO}_{2}$ concentrations do not affect the calculations of the air exchange rate by using this procedure, outside $\mathrm{CO}_{2}$ concentrations were not measured here. The spectral irradiance was continuously measured by a calibrated spectroradiometer (Ocean Optics, USA) equipped with a linear-array CCD detector and the measured values were converted into photon flux based on a well-established procedure by Madronich ${ }^{1}$, which was also used in a previous study. ${ }^{2}$ The photon flux values were used to estimate the photolysis frequency of HONO, J(HONO), which was about $8 \cdot 10^{-5} \mathrm{~s}^{-1}$ in the period from 9:30 to $11: 45$, and reached a maximum $1.5 \cdot 10^{-4}$ $\mathrm{s}^{-1}$ at noon. For more details about the estimations of $\mathrm{OH}$ radicals values readers are referred to Liu et al. ${ }^{3}$

Cooking emissions without pretreatment were introduced into the SESI chamber in real time through a $6.7-\mathrm{m}$ long Teflon tube (I.D. $4 \mathrm{~mm}$ ) with a flow rate of $1.4 \mathrm{~L} \mathrm{~min}^{-1}$, it should be noted that the flow rate decreased to $1.0 \mathrm{~L} \mathrm{~min}^{-1}$ at the sampling site that was close to the emission source. In order to assess the delay in the transport of VOCs from the sampling site to SESIHRMS through the 6.7-m sampling tube, we applied the headspace air of standard acetone-D7 (99.96\%, Sigma-Aldrich, USA) at the inlet of the sampling tube. It took less than $6 \mathrm{~s}$ for acetone-D7 to be detected, suggesting that the delay in the transport of VOCs is acceptable and temporal evolutions of primary and secondary VOCs can be properly recorded. 
51 Two sets of standard calibration solutions were used to calibrate the HRMS, to ensure that 52 reproducible sensitivity, stability and mass accuracy specified by the manufacturer were 53 achieved before individual measurement. One set of the standard solution was used for the 54 positive ion detection mode, containing n-Butylamine (0.0005\%), Caffeine (20 $\mu \mathrm{g})$, Ultramark $551621(0.001 \%)$ in a solution of $50 \%$ Acetonitrile/ 25\% Methanol/ 24\% Water/ 1\% Acetic Acid 56 per $10 \mathrm{~mL}$ of solution. The other set was for negative ion detection mode, containing Sodium 57 Dodecyl Sulfate $(29 \mu \mathrm{g})$, Sodium Taurocholate $(54 \mu \mathrm{g})$, and Ultramark $1621(0.001 \%)$ in a 58 solution of $50 \%$ Acetonitrile/ 25\% Methanol/ 24\% Water/ 1\% Acetic Acid per $10 \mathrm{~mL}$ of 59 solution.

60 Data analysis. The $\mathrm{m} / \mathrm{z}$ values, signal intensities and time points of all the ions detected were 61 extracted from the raw files and imported into a data matrix by using BreathFinder 62 (https://github.com/WanyangSun/BreathFinder). The signal intensity threshold to extract ions was set to $1 \times 10^{5}$ a.u. to ensure that the signal results from a compound instead of instrument 64 noises. 
Table S1: Detection limit, sensitivity of SESI-HRMS, and sampling efficiency of 6.7-m sampling tube.

\begin{tabular}{cccccc}
\hline \multirow{2}{*}{ Compounds } & Formula & $\begin{array}{c}\text { Detection } \\
\text { limit (ppb) }\end{array}$ & $\begin{array}{c}\text { Sensitivity } \\
\text { (a.u./ppb) }\end{array}$ & \multicolumn{2}{c}{ Sampling efficiency (\%)* } \\
\cline { 5 - 6 } & $\mathrm{C}_{4} \mathrm{H}_{8} \mathrm{O}_{2}$ & 0.14 & $8.12 \mathrm{Ep} 5$ & 2.9 & $\mathbf{2 0}$ ppb \\
\hline Ethyl acetate & 0.05 & $5.42 \mathrm{E} 5$ & 28.4 & 49.3 \\
Tert butyl methyl ether & $\mathrm{C}_{5} \mathrm{H}_{12} \mathrm{O}$ & 0.01 & $5 \mathrm{E} 6$ & 11.65 & 24.71 \\
\cline { 5 - 6 } & $\mathrm{C}_{6} \mathrm{H}_{12} \mathrm{O}$ & 0.01 &
\end{tabular}

*: Sampling efficiency is calculate by compare the signal intensity of the same concentration of gas standards detected by SESI-HRMS with the long sampling tube $(\sim 6.7 \mathrm{~m})$ and short sampling tube $(\sim 0.5$ $\mathrm{m})$.

Table S2. Detailed information of ions classified in Group 1 in Figure $1 .^{4-12}$

\begin{tabular}{|c|c|c|c|c|}
\hline$m / z$ & Composition & $\Delta \mathbf{m m u}$ & Ion mode & Compounds \\
\hline 95.0608 & $\mathrm{C} 5 \mathrm{H} 7 \mathrm{~N} 2$ & 0.39 & {$[\mathrm{M}+\mathrm{H}]^{+}$} & Methylpyrazine \\
\hline 109.0763 & C6H9N2 & 0.22 & {$[\mathrm{M}+\mathrm{H}]^{+}$} & Dimethylpyrazine \\
\hline 110.0602 & C6H8ON & 0.21 & {$[\mathrm{M}+\mathrm{H}]^{+}$} & 3-methoxy-4-pyridine \\
\hline 110.0795 & I & I & / & 1 \\
\hline 112.0759 & C6H10ON & 0.17 & {$[\mathrm{M}+\mathrm{H}]^{+}$} & l \\
\hline 121.0761 & C7H9N2 & 0.06 & {$[\mathrm{M}+\mathrm{H}]^{+}$} & 2-vinyl-6-methylpyrazine \\
\hline 122.0601 & $\mathrm{C} 7 \mathrm{H} 8 \mathrm{ON}$ & 0.06 & {$[\mathrm{M}+\mathrm{H}]^{+}$} & 1 \\
\hline 123.0635 & l & l & / & l \\
\hline 123.0917 & C7H11N2 & 0.04 & {$[\mathrm{M}+\mathrm{H}]^{+}$} & Trimethylpyrazine \\
\hline 124.0951 & H10ON7 & 0.93 & {$[\mathrm{M}+\mathrm{H}]^{+}$} & 1 \\
\hline 125.0710 & C6H9ON2 & 0 & {$[\mathrm{M}+\mathrm{H}]^{+}$} & 5-methyl-2-pyrazinemethanol \\
\hline 126.0914 & C7H12ON & -0.01 & {$[\mathrm{M}+\mathrm{H}]^{+}$} & I \\
\hline 127.0390 & C6H7O3 & 0 & {$[\mathrm{M}+\mathrm{H}]^{+}$} & l \\
\hline 128.0423 & H2N9 & -0.44 & {$[\mathrm{M}+\mathrm{H}]^{+}$} & I \\
\hline 128.1070 & C7H14ON & -0.01 & {$[\mathrm{M}+\mathrm{H}]^{+}$} & $\mathrm{N}$-acetylpiperidine \\
\hline 135.0440 & $\mathrm{C} 8 \mathrm{H} 7 \mathrm{O} 2$ & -0.05 & {$[\mathrm{M}+\mathrm{H}]^{+}$} & 1 \\
\hline 135.0916 & C8H11N2 & -0.08 & {$[\mathrm{M}+\mathrm{H}]^{+}$} & 2-methyl-5-propenylpyrazine \\
\hline 136.0950 & CH10ON7 & 0.83 & {$[\mathrm{M}+\mathrm{H}]^{+}$} & 1 \\
\hline 137.0707 & C7H9ON2 & -0.17 & {$[\mathrm{M}+\mathrm{H}]^{+}$} & 2-acetyl-3-methylpyrazine \\
\hline 137.1072 & C8H13N2 & -0.13 & {$[\mathrm{M}+\mathrm{H}]^{+}$} & 2,5-dimethyl-3-ethylpyrazine \\
\hline 138.0547 & $\mathrm{C} 7 \mathrm{H} 8 \mathrm{O} 2 \mathrm{~N}$ & -0.2 & {$[\mathrm{M}+\mathrm{H}]^{+}$} & Methylpyridine-3-carboxylate \\
\hline 138.1106 & CH12ON7 & 0.86 & {$[\mathrm{M}+\mathrm{H}]^{+}$} & 1 \\
\hline 149.1069 & C9H13N2 & -0.36 & {$[\mathrm{M}+\mathrm{H}]^{+}$} & 1 \\
\hline 151.1227 & $\mathrm{C} 9 \mathrm{H} 15 \mathrm{~N} 2$ & -0.33 & {$[\mathrm{M}+\mathrm{H}]^{+}$} & 2,6-diethyl-3-methylpyrazine \\
\hline 152.0704 & $\mathrm{C} 8 \mathrm{H} 10 \mathrm{O} 2 \mathrm{~N}$ & -0.25 & {$[\mathrm{M}+\mathrm{H}]^{+}$} & 1 \\
\hline 168.1128 & $\mathrm{C} 8 \mathrm{H} 14 \mathrm{ON} 3$ & -0.33 & {$[\mathrm{M}+\mathrm{H}]^{+}$} & l \\
\hline
\end{tabular}




\begin{tabular}{|c|c|c|c|c|}
\hline 179.1540 & $\mathrm{C} 11 \mathrm{H} 19 \mathrm{~N} 2$ & -0.37 & {$[\mathrm{M}+\mathrm{H}]^{+}$} & I \\
\hline 182.1285 & C9H16ON3 & -0.28 & {$[\mathrm{M}+\mathrm{H}]^{+}$} & I \\
\hline 183.1126 & $\mathrm{C} 9 \mathrm{H} 15 \mathrm{O} 2 \mathrm{~N} 2$ & -0.27 & {$[\mathrm{M}+\mathrm{H}]^{+}$} & I \\
\hline 185.1283 & C9H17O2N2 & -0.32 & {$[\mathrm{M}+\mathrm{H}]^{+}$} & l \\
\hline 189.1594 & $\mathrm{C} 9 \mathrm{H} 21 \mathrm{O} 2 \mathrm{~N} 2$ & -0.37 & {$[\mathrm{M}+\mathrm{H}]^{+}$} & I \\
\hline 195.1125 & $\mathrm{C} 10 \mathrm{H} 15 \mathrm{O} 2 \mathrm{~N} 2$ & -0.34 & {$[\mathrm{M}+\mathrm{H}]^{+}$} & I \\
\hline 196.1158 & $\mathrm{C} 3 \mathrm{H} 14 \mathrm{O} 3 \mathrm{~N} 7$ & 0.54 & {$[\mathrm{M}+\mathrm{H}]^{+}$} & I \\
\hline 196.1441 & C10H18ON3 & -0.37 & {$[\mathrm{M}+\mathrm{H}]^{+}$} & I \\
\hline 199.1437 & $\mathrm{C} 10 \mathrm{H} 19 \mathrm{O} 2 \mathrm{~N} 2$ & -0.4 & {$[\mathrm{M}+\mathrm{H}]^{+}$} & I \\
\hline 200.0913 & C9H14O4N & -0.39 & {$[\mathrm{M}+\mathrm{H}]^{+}$} & I \\
\hline 201.0948 & C2H13O5N6 & 0.48 & {$[\mathrm{M}+\mathrm{H}]^{+}$} & I \\
\hline 208.0964 & $\mathrm{C} 11 \mathrm{H} 14 \mathrm{O} 3 \mathrm{~N}$ & -0.37 & {$[\mathrm{M}+\mathrm{H}]^{+}$} & I \\
\hline 208.1438 & C11H18ON3 & -0.42 & {$[\mathrm{M}+\mathrm{H}]^{+}$} & I \\
\hline 210.1597 & $\mathrm{C} 11 \mathrm{H} 20 \mathrm{ON} 3$ & -0.43 & {$[\mathrm{M}+\mathrm{H}]^{+}$} & l \\
\hline 211.1073 & $\mathrm{C} 10 \mathrm{H} 15 \mathrm{O} 3 \mathrm{~N} 2$ & -0.45 & {$[\mathrm{M}+\mathrm{H}]^{+}$} & I \\
\hline 61.9738 & I & / & I & I \\
\hline 62.0002 & I & / & I & l \\
\hline 62.9840 & I & / & I & l \\
\hline 62.9913 & I & I & I & I \\
\hline 63.9611 & I & I & I & I \\
\hline 63.9912 & I & / & I & I \\
\hline 79.9560 & I & / & I & l \\
\hline 80.9638 & I & I & I & I \\
\hline 92.9928 & I & / & I & l \\
\hline 94.9846 & I & / & I & I \\
\hline 95.9509 & I & / & I & I \\
\hline 95.9925 & I & / & I & I \\
\hline 96.9587 & I & I & I & l \\
\hline 102.0183 & $\mathrm{C} 3 \mathrm{H} 4 \mathrm{O} 3 \mathrm{~N}$ & -1.37 & {$[\mathrm{M}-\mathrm{H}]^{-}$} & I \\
\hline 111.9459 & I & I & I & I \\
\hline 111.9874 & I & / & I & I \\
\hline 112.9537 & I & I & I & l \\
\hline 119.0489 & $\mathrm{C} 8 \mathrm{H} 7 \mathrm{O}$ & -1.3 & {$[\mathrm{M}-\mathrm{H}]^{-}$} & I \\
\hline 120.0523 & CH6O2N5 & -0.4 & {$[\mathrm{M}-\mathrm{H}]^{-}$} & I \\
\hline 121.0282 & C7H5O2 & -1.29 & {$[\mathrm{M}-\mathrm{H}]^{-}$} & I \\
\hline 122.0315 & I & I & I & I \\
\hline 123.9875 & $\mathrm{CH} 2 \mathrm{O} 6 \mathrm{~N}$ & -1.28 & {$[\mathrm{M}-\mathrm{H}]^{-}$} & I \\
\hline 124.0391 & $\mathrm{C} 6 \mathrm{H} 6 \mathrm{O} 2 \mathrm{~N}$ & -1.32 & {$[\mathrm{M}-\mathrm{H}]^{-}$} & I \\
\hline 124.9827 & I & I & I & I \\
\hline 134.0361 & C6H4ON3 & 0.11 & {$[\mathrm{M}-\mathrm{H}]^{-}$} & I \\
\hline 135.0440 & $\mathrm{C} 8 \mathrm{H} 7 \mathrm{O} 2$ & -1.21 & {$[\mathrm{M}-\mathrm{H}]^{-}$} & / \\
\hline 136.0154 & $\mathrm{C} 5 \mathrm{H} 2 \mathrm{O} 2 \mathrm{~N} 3$ & 0.13 & {$[\mathrm{M}-\mathrm{H}]^{-}$} & l \\
\hline 136.0473 & CH6O3N5 & -0.35 & {$[\mathrm{M}-\mathrm{H}]^{-}$} & I \\
\hline 137.0232 & $\mathrm{C} 7 \mathrm{H} 5 \mathrm{O} 3$ & -1.23 & {$[\mathrm{M}-\mathrm{H}]^{-}$} & 1 \\
\hline
\end{tabular}




\begin{tabular}{|c|c|c|c|c|}
\hline 140.9778 & I & I & I & I \\
\hline 141.0181 & C6H5O4 & -1.2 & {$[\mathrm{M}-\mathrm{H}]^{-}$} & I \\
\hline 141.9440 & I & I & I & I \\
\hline 149.0232 & C8H5O3 & -1.16 & {$[\mathrm{M}-\mathrm{H}]^{-}$} & I \\
\hline 149.0597 & С9H9O2 & -1.18 & {$[\mathrm{M}-\mathrm{H}]^{-}$} & / \\
\hline 150.0311 & $\mathrm{C} 6 \mathrm{H} 4 \mathrm{O} 2 \mathrm{~N} 3$ & 0.17 & {$[\mathrm{M}-\mathrm{H}]^{-}$} & / \\
\hline 151.0389 & $\mathrm{C} 8 \mathrm{H} 7 \mathrm{O} 3$ & -1.15 & {$[\mathrm{M}-\mathrm{H}]^{-}$} & / \\
\hline 152.0422 & CH6O4N5 & -0.26 & {$[\mathrm{M}-\mathrm{H}]^{-}$} & / \\
\hline 152.0467 & C6H6O2N3 & 0.17 & {$[\mathrm{M}-\mathrm{H}]^{-}$} & / \\
\hline 153.0181 & C7H5O4 & -1.14 & {$[\mathrm{M}-\mathrm{H}]^{-}$} & / \\
\hline 164.0343 & $\mathrm{C} 8 \mathrm{H} 6 \mathrm{O} 3 \mathrm{~N}$ & -1.07 & {$[\mathrm{M}-\mathrm{H}]^{-}$} & / \\
\hline 165.0184 & C8H5O4 & -1.03 & {$[\mathrm{M}-\mathrm{H}]^{-}$} & / \\
\hline 166.0498 & $\mathrm{C} 8 \mathrm{H} 8 \mathrm{O} 3 \mathrm{~N}$ & -1.11 & {$[\mathrm{M}-\mathrm{H}]^{-}$} & / \\
\hline 167.0338 & $\mathrm{C} 8 \mathrm{H} 7 \mathrm{O} 4$ & -1.05 & {$[\mathrm{M}-\mathrm{H}]^{-}$} & / \\
\hline 168.0292 & $\mathrm{C} 7 \mathrm{H} 6 \mathrm{O} 4 \mathrm{~N}$ & -1 & {$[\mathrm{M}-\mathrm{H}]^{-}$} & / \\
\hline 179.9881 & I & I & I & / \\
\hline 181.0497 & C9H9O4 & -0.98 & {$[\mathrm{M}-\mathrm{H}]^{-}$} & / \\
\hline 182.0449 & $\mathrm{C} 8 \mathrm{H} 8 \mathrm{O} 4 \mathrm{~N}$ & -0.94 & {$[\mathrm{M}-\mathrm{H}]^{-}$} & / \\
\hline 184.0242 & C7H6O5N & -0.98 & {$[\mathrm{M}-\mathrm{H}]^{-}$} & I \\
\hline 194.0449 & $\mathrm{C} 9 \mathrm{H} 8 \mathrm{O} 4 \mathrm{~N}$ & -0.91 & {$[\mathrm{M}-\mathrm{H}]^{-}$} & / \\
\hline 198.0400 & $\mathrm{C} 8 \mathrm{H} 8 \mathrm{O} 5 \mathrm{~N}$ & -0.83 & {$[\mathrm{M}-\mathrm{H}]^{-}$} & / \\
\hline 279.9856 & $\mathrm{C} 9 \mathrm{H} 2 \mathrm{O} 8 \mathrm{~N} 3$ & 1.02 & {$[\mathrm{M}-\mathrm{H}]^{-}$} & / \\
\hline 295.9805 & $\mathrm{C} 9 \mathrm{H} 2 \mathrm{O} 9 \mathrm{~N} 3$ & 0.96 & {$[\mathrm{M}-\mathrm{H}]^{-}$} & 1 \\
\hline
\end{tabular}

Table S3. Detailed information of ions classified in Group 2 in Figure 1. ${ }^{4-12}$

\begin{tabular}{ccccc}
\hline $\boldsymbol{m} / \boldsymbol{z}$ & Composition & $\Delta \mathbf{m m u}$ & Ion mode & Compounds \\
\hline 70.0658 & C4H8N & 0.71 & {$[\mathrm{M}+\mathrm{H}]^{+}$} & $/$ \\
72.0451 & C3H6ON & 0.75 & {$[\mathrm{M}+\mathrm{H}]^{+}$} & $/$ \\
85.0653 & C5H9O & 0.53 & {$[\mathrm{M}+\mathrm{H}]^{+}$} & Pentenal \\
86.0606 & C4H8ON & 0.52 & {$[\mathrm{M}+\mathrm{H}]^{+}$} & $/$ \\
104.0709 & C4H10O2N & 0.27 & {$[\mathrm{M}+\mathrm{H}]^{+}$} & $/$ \\
111.0806 & C7H11O & 0.18 & {$[\mathrm{M}+\mathrm{H}]^{+}$} & Heptadienal \\
113.0599 & C6H9O2 & 0.17 & {$[\mathrm{M}+\mathrm{H}]^{+}$} & $/$ \\
113.0962 & C7H13O & 0.16 & {$[\mathrm{M}+\mathrm{H}]^{+}$} & Heptenal \\
116.1071 & C6H14ON & 0.11 & {$[\mathrm{M}+\mathrm{H}]^{+}$} & $/$ \\
124.0757 & C7H10ON & 0.04 & {$[\mathrm{M}+\mathrm{H}]^{+}$} & $/$ \\
125.0597 & C7H9O2 & -0.01 & {$[\mathrm{M}+\mathrm{H}]^{+}$} & 2-acetyl-5-methylfuran \\
126.0549 & C6H8O2N & -0.03 & {$[\mathrm{M}+\mathrm{H}]^{+}$} & $/$ \\
127.0753 & C7H11O2 & -0.02 & {$[\mathrm{M}+\mathrm{H}]^{+}$} & Ethylfuran-2-methanol \\
128.0706 & C6H10O2N & -0.02 & {$[\mathrm{M}+\mathrm{H}]^{+}$} & $/$ \\
130.0863 & C6H12O2N & -0.06 & {$[\mathrm{M}+\mathrm{H}]^{+}$} & $/$ \\
\hline
\end{tabular}




\begin{tabular}{|c|c|c|c|c|}
\hline 130.1226 & $\mathrm{C} 7 \mathrm{H} 16 \mathrm{ON}$ & -0.08 & {$[\mathrm{M}+\mathrm{H}]^{+}$} & I \\
\hline 135.1167 & $\mathrm{C} 10 \mathrm{H} 15$ & -0.1 & {$[\mathrm{M}+\mathrm{H}]^{+}$} & / \\
\hline 136.0756 & $\mathrm{C} 8 \mathrm{H} 10 \mathrm{ON}$ & -0.16 & {$[\mathrm{M}+\mathrm{H}]^{+}$} & I \\
\hline 137.5460 & I & I & I & I \\
\hline 138.0911 & $\mathrm{C} 8 \mathrm{H} 12 \mathrm{ON}$ & -0.14 & {$[\mathrm{M}+\mathrm{H}]^{+}$} & I \\
\hline 139.0865 & C7H11ON2 & -0.13 & {$[\mathrm{M}+\mathrm{H}]^{+}$} & I \\
\hline 140.0704 & $\mathrm{C} 7 \mathrm{H} 10 \mathrm{O} 2 \mathrm{~N}$ & -0.28 & {$[\mathrm{M}+\mathrm{H}]^{+}$} & / \\
\hline 140.1068 & C8H14ON & -0.18 & {$[\mathrm{M}+\mathrm{H}]^{+}$} & I \\
\hline 143.1182 & C7H15ON2 & -0.29 & {$[\mathrm{M}+\mathrm{H}]^{+}$} & I \\
\hline 144.1017 & $\mathrm{C} 7 \mathrm{H} 14 \mathrm{O} 2 \mathrm{~N}$ & -0.2 & {$[\mathrm{M}+\mathrm{H}]^{+}$} & / \\
\hline 145.0969 & C6H13O2N2 & -0.21 & {$[\mathrm{M}+\mathrm{H}]^{+}$} & I \\
\hline 150.0909 & C9H12ON & -0.36 & {$[\mathrm{M}+\mathrm{H}]^{+}$} & I \\
\hline 150.1274 & $\mathrm{C} 10 \mathrm{H} 16 \mathrm{~N}$ & -0.29 & {$[\mathrm{M}+\mathrm{H}]^{+}$} & I \\
\hline 151.0863 & C8H11ON2 & -0.29 & {$[\mathrm{M}+\mathrm{H}]^{+}$} & / \\
\hline 153.1018 & C8H13ON2 & -0.33 & {$[\mathrm{M}+\mathrm{H}]^{+}$} & I \\
\hline 153.1271 & $\mathrm{C} 10 \mathrm{H} 17 \mathrm{O}$ & -0.26 & {$[\mathrm{M}+\mathrm{H}]^{+}$} & Decadienal \\
\hline 154.0858 & $\mathrm{C} 8 \mathrm{H} 12 \mathrm{O} 2 \mathrm{~N}$ & -0.43 & {$[\mathrm{M}+\mathrm{H}]^{+}$} & I \\
\hline 154.0940 & $\mathrm{C} 2 \mathrm{H} 12 \mathrm{O} 3 \mathrm{~N} 5$ & 0.48 & {$[\mathrm{M}+\mathrm{H}]^{+}$} & / \\
\hline 154.1304 & I & I & I & / \\
\hline 156.1380 & C9H18ON & -0.34 & {$[\mathrm{M}+\mathrm{H}]^{+}$} & / \\
\hline 158.0810 & $\mathrm{C} 7 \mathrm{H} 12 \mathrm{O} 3 \mathrm{~N}$ & -0.19 & {$[\mathrm{M}+\mathrm{H}]^{+}$} & / \\
\hline 159.1126 & $\mathrm{C} 7 \mathrm{H} 15 \mathrm{O} 2 \mathrm{~N} 2$ & -0.26 & {$[\mathrm{M}+\mathrm{H}]^{+}$} & / \\
\hline 161.0707 & C9H9ON2 & -0.19 & {$[\mathrm{M}+\mathrm{H}]^{+}$} & / \\
\hline 164.1068 & C10H14ON & -0.24 & {$[\mathrm{M}+\mathrm{H}]^{+}$} & / \\
\hline 165.1020 & $\mathrm{C} 9 \mathrm{H} 13 \mathrm{ON} 2$ & -0.22 & {$[\mathrm{M}+\mathrm{H}]^{+}$} & I \\
\hline 167.1175 & C9H15ON2 & -0.33 & {$[\mathrm{M}+\mathrm{H}]^{+}$} & / \\
\hline 172.0964 & $\mathrm{C} 8 \mathrm{H} 14 \mathrm{O} 3 \mathrm{~N}$ & -0.39 & {$[\mathrm{M}+\mathrm{H}]^{+}$} & / \\
\hline 173.1283 & $\mathrm{C} 8 \mathrm{H} 17 \mathrm{O} 2 \mathrm{~N} 2$ & -0.27 & {$[\mathrm{M}+\mathrm{H}]^{+}$} & / \\
\hline 174.1314 & I & I & I & / \\
\hline 175.0864 & $\mathrm{C} 10 \mathrm{H} 11 \mathrm{ON} 2$ & -0.29 & {$[\mathrm{M}+\mathrm{H}]^{+}$} & / \\
\hline 179.1176 & $\mathrm{C} 10 \mathrm{H} 15 \mathrm{ON} 2$ & -0.37 & {$[\mathrm{M}+\mathrm{H}]^{+}$} & / \\
\hline 181.0968 & $\mathrm{C} 9 \mathrm{H} 13 \mathrm{O} 2 \mathrm{~N} 2$ & -0.38 & {$[\mathrm{M}+\mathrm{H}]^{+}$} & / \\
\hline 182.1537 & $\mathrm{C} 11 \mathrm{H} 20 \mathrm{ON}$ & -0.29 & {$[\mathrm{M}+\mathrm{H}]^{+}$} & / \\
\hline 186.1121 & C9H16O3N & -0.32 & {$[\mathrm{M}+\mathrm{H}]^{+}$} & / \\
\hline 189.1019 & C11H13ON2 & -0.37 & {$[\mathrm{M}+\mathrm{H}]^{+}$} & / \\
\hline 191.1427 & C13H19O & -0.3 & {$[\mathrm{M}+\mathrm{H}]^{+}$} & / \\
\hline 197.1282 & $\mathrm{C} 10 \mathrm{H} 17 \mathrm{O} 2 \mathrm{~N} 2$ & -0.38 & {$[\mathrm{M}+\mathrm{H}]^{+}$} & / \\
\hline 200.1276 & $\mathrm{C} 10 \mathrm{H} 18 \mathrm{O} 3 \mathrm{~N}$ & -0.41 & {$[\mathrm{M}+\mathrm{H}]^{+}$} & I \\
\hline 201.1593 & $\mathrm{C} 10 \mathrm{H} 21 \mathrm{O} 2 \mathrm{~N} 2$ & -0.44 & {$[\mathrm{M}+\mathrm{H}]^{+}$} & / \\
\hline 209.1280 & $\mathrm{C} 11 \mathrm{H} 17 \mathrm{O} 2 \mathrm{~N} 2$ & -0.39 & {$[\mathrm{M}+\mathrm{H}]^{+}$} & / \\
\hline 224.1641 & $\mathrm{C} 13 \mathrm{H} 22 \mathrm{O} 2 \mathrm{~N}$ & -0.47 & {$[\mathrm{M}+\mathrm{H}]^{+}$} & / \\
\hline 226.1797 & $\mathrm{C} 13 \mathrm{H} 24 \mathrm{O} 2 \mathrm{~N}$ & -0.5 & {$[\mathrm{M}+\mathrm{H}]^{+}$} & / \\
\hline 227.1830 & I & I & I & / \\
\hline 237.1844 & $\mathrm{C} 15 \mathrm{H} 25 \mathrm{O} 2$ & -0.47 & {$[\mathrm{M}+\mathrm{H}]^{+}$} & I \\
\hline
\end{tabular}




\begin{tabular}{|c|c|c|c|c|}
\hline 305.2466 & $\mathrm{C} 20 \mathrm{H} 33 \mathrm{O} 2$ & -0.78 & {$[\mathrm{M}+\mathrm{H}]^{+}$} & I \\
\hline 306.2501 & C13H32O3N5 & 0.16 & {$[\mathrm{M}+\mathrm{H}]^{+}$} & l \\
\hline 65.9983 & l & / & I & / \\
\hline 78.9897 & I & I & I & I \\
\hline 79.9975 & I & / & I & I \\
\hline 85.0281 & I & I & I & I \\
\hline 88.0026 & $\mathrm{C} 2 \mathrm{H} 2 \mathrm{O} 3 \mathrm{~N}$ & -1.4 & {$[\mathrm{M}-\mathrm{H}]^{-}$} & l \\
\hline 88.9866 & C2HO4 & -1.39 & {$[\mathrm{M}-\mathrm{H}]^{-}$} & I \\
\hline 91.0023 & $\mathrm{C} 2 \mathrm{H} 3 \mathrm{O} 4$ & -1.39 & {$[\mathrm{M}-\mathrm{H}]^{-}$} & I \\
\hline 91.9975 & $\mathrm{CH} 2 \mathrm{O} 4 \mathrm{~N}$ & -1.4 & {$[\mathrm{M}-\mathrm{H}]^{-}$} & I \\
\hline 94.9972 & СH3O5 & -1.41 & {$[\mathrm{M}-\mathrm{H}]^{-}$} & I \\
\hline 97.9881 & $\mathrm{C} 3 \mathrm{O} 3 \mathrm{~N}$ & -0.24 & {$[\mathrm{M}-\mathrm{H}]^{-}$} & I \\
\hline 106.0132 & $\mathrm{C} 2 \mathrm{H} 4 \mathrm{O} 4 \mathrm{~N}$ & -1.35 & {$[\mathrm{M}-\mathrm{H}]^{-}$} & I \\
\hline 106.9973 & $\mathrm{C} 2 \mathrm{H} 3 \mathrm{O} 5$ & -1.34 & {$[\mathrm{M}-\mathrm{H}]^{-}$} & I \\
\hline 107.9925 & I & I & I & l \\
\hline 108.9878 & I & I & I & I \\
\hline 116.0341 & $\mathrm{C} 4 \mathrm{H} 6 \mathrm{O} 3 \mathrm{~N}$ & -1.29 & {$[\mathrm{M}-\mathrm{H}]^{-}$} & I \\
\hline 123.0074 & $\mathrm{C} 6 \mathrm{H} 3 \mathrm{O} 3$ & -1.29 & {$[\mathrm{M}-\mathrm{H}]^{-}$} & I \\
\hline 127.0024 & $\mathrm{C} 5 \mathrm{H} 3 \mathrm{O} 4$ & -1.29 & {$[\mathrm{M}-\mathrm{H}]^{-}$} & I \\
\hline 131.0337 & $\mathrm{C} 5 \mathrm{H} 7 \mathrm{O} 4$ & -1.24 & {$[\mathrm{M}-\mathrm{H}]^{-}$} & l \\
\hline 138.0185 & $\mathrm{C} 6 \mathrm{H} 4 \mathrm{O} 3 \mathrm{~N}$ & -1.21 & {$[\mathrm{M}-\mathrm{H}]^{-}$} & I \\
\hline 139.0025 & C6H3O4 & -1.21 & {$[\mathrm{M}-\mathrm{H}]^{-}$} & I \\
\hline 139.0389 & $\mathrm{C} 7 \mathrm{H} 7 \mathrm{O} 3$ & -1.23 & {$[\mathrm{M}-\mathrm{H}]^{-}$} & I \\
\hline 143.0337 & $\mathrm{C} 6 \mathrm{H} 7 \mathrm{O} 4$ & -1.19 & {$[\mathrm{M}-\mathrm{H}]^{-}$} & I \\
\hline 145.0131 & C5H5O5 & -1.19 & {$[\mathrm{M}-\mathrm{H}]^{-}$} & I \\
\hline 152.0342 & $\mathrm{C} 7 \mathrm{H} 6 \mathrm{O} 3 \mathrm{~N}$ & -1.16 & {$[\mathrm{M}-\mathrm{H}]^{-}$} & I \\
\hline 156.9727 & I & I & I & I \\
\hline 157.0132 & C6H5O5 & -1.1 & {$[\mathrm{M}-\mathrm{H}]^{-}$} & I \\
\hline 159.0288 & C6H7O5 & -1.1 & {$[\mathrm{M}-\mathrm{H}]^{-}$} & I \\
\hline 164.0190 & $\mathrm{C} 4 \mathrm{H} 6 \mathrm{O} 6 \mathrm{~N}$ & -1.12 & {$[\mathrm{M}-\mathrm{H}]^{-}$} & I \\
\hline 171.0288 & $\mathrm{C} 7 \mathrm{H} 7 \mathrm{O} 5$ & -0.99 & {$[\mathrm{M}-\mathrm{H}]^{-}$} & / \\
\hline 184.0970 & $\mathrm{C} 9 \mathrm{H} 14 \mathrm{O} 3 \mathrm{~N}$ & -0.94 & {$[\mathrm{M}-\mathrm{H}]^{-}$} & I \\
\hline 184.9833 & C5HO6N2 & -0.7 & {$[\mathrm{M}-\mathrm{H}]^{-}$} & I \\
\hline 185.0445 & C8H9O5 & -0.95 & {$[\mathrm{M}-\mathrm{H}]^{-}$} & / \\
\hline 196.9834 & C6HO6N2 & -0.62 & {$[\mathrm{M}-\mathrm{H}]^{-}$} & I \\
\hline 208.0454 & $\mathrm{C} 6 \mathrm{H} 10 \mathrm{O} 7 \mathrm{~N}$ & -0.8 & {$[\mathrm{M}-\mathrm{H}]^{-}$} & I \\
\hline 262.9756 & I & I & I & / \\
\hline 312.9725 & $\mathrm{C} 13 \mathrm{HO} 8 \mathrm{~N} 2$ & -1.42 & {$[\mathrm{M}-\mathrm{H}]^{-}$} & I \\
\hline
\end{tabular}

Table S4. Detailed information of ions classified in Group 3 in Figure 1. ${ }^{4-12}$

\begin{tabular}{ccccc}
\hline$m / z$ & Composition & $\Delta \mathrm{mmu}$ & Ion mode & Compounds \\
\hline
\end{tabular}




\begin{tabular}{|c|c|c|c|c|}
\hline 87.0809 & $\mathrm{C} 5 \mathrm{H} 11 \mathrm{O}$ & 0.52 & {$[\mathrm{M}+\mathrm{H}]^{+}$} & Pentanal \\
\hline 90.0918 & $\mathrm{C} 4 \mathrm{H} 12 \mathrm{ON}$ & 0.46 & {$[\mathrm{M}+\mathrm{H}]^{+}$} & I \\
\hline 100.0760 & C5H10ON & 0.33 & {$[\mathrm{M}+\mathrm{H}]^{+}$} & I \\
\hline 101.0600 & $\mathrm{C} 5 \mathrm{H} 9 \mathrm{O} 2$ & 0.32 & {$[\mathrm{M}+\mathrm{H}]^{+}$} & I \\
\hline 103.0757 & C5H11O2 & 0.3 & {$[\mathrm{M}+\mathrm{H}]^{+}$} & I \\
\hline 105.0913 & $\mathrm{C} 5 \mathrm{H} 13 \mathrm{O} 2$ & 0.27 & {$[\mathrm{M}+\mathrm{H}]^{+}$} & I \\
\hline 118.0864 & $\mathrm{C} 5 \mathrm{H} 12 \mathrm{O} 2 \mathrm{~N}$ & 0.09 & {$[\mathrm{M}+\mathrm{H}]^{+}$} & I \\
\hline 121.1013 & C9H13 & 0.06 & {$[\mathrm{M}+\mathrm{H}]^{+}$} & I \\
\hline 125.0961 & $\mathrm{C} 8 \mathrm{H} 13 \mathrm{O}$ & 0.02 & {$[\mathrm{M}+\mathrm{H}]^{+}$} & Octadienal \\
\hline 126.0994 & C6H12N3 & -3.14 & {$[\mathrm{M}+\mathrm{H}]^{+}$} & I \\
\hline 127.1117 & С8H15O & 0 & {$[\mathrm{M}+\mathrm{H}]^{+}$} & Octenal \\
\hline 129.0910 & $\mathrm{C} 7 \mathrm{H} 13 \mathrm{O} 2$ & -0.02 & {$[\mathrm{M}+\mathrm{H}]^{+}$} & 5-propyldihydro-2(3H)-furanone \\
\hline 131.1065 & $\mathrm{C} 7 \mathrm{H} 15 \mathrm{O} 2$ & -0.1 & {$[\mathrm{M}+\mathrm{H}]^{+}$} & I \\
\hline 132.1018 & $\mathrm{C} 6 \mathrm{H} 14 \mathrm{O} 2 \mathrm{~N}$ & -0.09 & {$[\mathrm{M}+\mathrm{H}]^{+}$} & I \\
\hline 137.0959 & С9H13O & -0.18 & {$[\mathrm{M}+\mathrm{H}]^{+}$} & Nonatrienal \\
\hline 139.1115 & С9H15O & -0.2 & {$[\mathrm{M}+\mathrm{H}]^{+}$} & Nonadienal \\
\hline 140.1150 & I & I & 1 & I \\
\hline 141.0908 & $\mathrm{C} 8 \mathrm{H} 13 \mathrm{O} 2$ & -0.21 & {$[\mathrm{M}+\mathrm{H}]^{+}$} & I \\
\hline 142.0862 & $\mathrm{C} 7 \mathrm{H} 12 \mathrm{O} 2 \mathrm{~N}$ & -0.07 & {$[\mathrm{M}+\mathrm{H}]^{+}$} & I \\
\hline 142.0942 & I & I & I & I \\
\hline 143.1066 & $\mathrm{C} 8 \mathrm{H} 15 \mathrm{O} 2$ & -0.08 & {$[\mathrm{M}+\mathrm{H}]^{+}$} & I \\
\hline 144.0477 & I & I & 1 & I \\
\hline 144.0655 & $\mathrm{C} 6 \mathrm{H} 10 \mathrm{O} 3 \mathrm{~N}$ & -0.04 & {$[\mathrm{M}+\mathrm{H}]^{+}$} & I \\
\hline 144.1098 & I & I & I & I \\
\hline 145.0512 & C7H5N4 & 0.3 & {$[\mathrm{M}+\mathrm{H}]^{+}$} & I \\
\hline 146.0438 & $\mathrm{CH} 4 \mathrm{O} 2 \mathrm{~N} 7$ & 1.3 & {$[\mathrm{M}+\mathrm{H}]^{+}$} & I \\
\hline 146.1175 & $\mathrm{C} 7 \mathrm{H} 16 \mathrm{O} 2 \mathrm{~N}$ & -0.04 & {$[\mathrm{M}+\mathrm{H}]^{+}$} & I \\
\hline 151.1116 & C10H15O & -0.15 & {$[\mathrm{M}+\mathrm{H}]^{+}$} & I \\
\hline 152.1150 & $\mathrm{C} 3 \mathrm{H} 14 \mathrm{O} 2 \mathrm{~N} 5$ & 0.62 & {$[\mathrm{M}+\mathrm{H}]^{+}$} & I \\
\hline 154.0494 & $\mathrm{C} 7 \mathrm{H} 8 \mathrm{O} 3 \mathrm{~N}$ & -0.38 & {$[\mathrm{M}+\mathrm{H}]^{+}$} & I \\
\hline 154.1222 & $\mathrm{C} 9 \mathrm{H} 16 \mathrm{ON}$ & -0.41 & {$[\mathrm{M}+\mathrm{H}]^{+}$} & I \\
\hline 156.1017 & $\mathrm{C} 8 \mathrm{H} 14 \mathrm{O} 2 \mathrm{~N}$ & -0.22 & {$[\mathrm{M}+\mathrm{H}]^{+}$} & I \\
\hline 157.1221 & $\mathrm{C} 9 \mathrm{H} 17 \mathrm{O} 2$ & -0.21 & {$[\mathrm{M}+\mathrm{H}]^{+}$} & I \\
\hline 158.1254 & I & I & I & I \\
\hline 160.1330 & $\mathrm{C} 8 \mathrm{H} 18 \mathrm{O} 2 \mathrm{~N}$ & -0.27 & {$[\mathrm{M}+\mathrm{H}]^{+}$} & I \\
\hline 162.0910 & $\mathrm{C} 10 \mathrm{H} 12 \mathrm{ON}$ & -0.36 & {$[\mathrm{M}+\mathrm{H}]^{+}$} & I \\
\hline 162.1486 & $\mathrm{C} 8 \mathrm{H} 20 \mathrm{O} 2 \mathrm{~N}$ & -0.31 & {$[\mathrm{M}+\mathrm{H}]^{+}$} & I \\
\hline 165.0908 & $\mathrm{C} 10 \mathrm{H} 13 \mathrm{O} 2$ & -0.18 & {$[\mathrm{M}+\mathrm{H}]^{+}$} & I \\
\hline 165.1271 & $\mathrm{C} 11 \mathrm{H} 17 \mathrm{O}$ & -0.2 & {$[\mathrm{M}+\mathrm{H}]^{+}$} & I \\
\hline 167.0700 & C9H11O3 & -0.24 & {$[\mathrm{M}+\mathrm{H}]^{+}$} & I \\
\hline 167.1063 & $\mathrm{C} 10 \mathrm{H} 15 \mathrm{O} 2$ & -0.3 & {$[\mathrm{M}+\mathrm{H}]^{+}$} & I \\
\hline 167.1428 & C11H19O & -0.23 & {$[\mathrm{M}+\mathrm{H}]^{+}$} & I \\
\hline 168.1097 & C3H14O3N5 & 0.58 & {$[\mathrm{M}+\mathrm{H}]^{+}$} & I \\
\hline 169.1220 & $\mathrm{C} 10 \mathrm{H} 17 \mathrm{O} 2$ & -0.32 & {$[\mathrm{M}+\mathrm{H}]^{+}$} & 1 \\
\hline
\end{tabular}




\begin{tabular}{|c|c|c|c|c|}
\hline 170.1172 & $\mathrm{C} 9 \mathrm{H} 16 \mathrm{O} 2 \mathrm{~N}$ & -0.34 & {$[\mathrm{M}+\mathrm{H}]^{+}$} & I \\
\hline 170.1252 & I & l & l & I \\
\hline 170.1536 & $\mathrm{C} 10 \mathrm{H} 20 \mathrm{ON}$ & -0.3 & {$[\mathrm{M}+\mathrm{H}]^{+}$} & l \\
\hline 171.1377 & $\mathrm{C} 10 \mathrm{H} 19 \mathrm{O} 2$ & -0.31 & {$[\mathrm{M}+\mathrm{H}]^{+}$} & 5-pentyldihydro-2(3H)-furanone \\
\hline 171.1569 & l & I & l & / \\
\hline 172.1328 & $\mathrm{C} 9 \mathrm{H} 18 \mathrm{O} 2 \mathrm{~N}$ & -0.34 & {$[\mathrm{M}+\mathrm{H}]^{+}$} & / \\
\hline 172.1693 & $\mathrm{C} 10 \mathrm{H} 22 \mathrm{ON}$ & -0.4 & {$[\mathrm{M}+\mathrm{H}]^{+}$} & I \\
\hline 173.1363 & l & / & / & / \\
\hline 174.1486 & $\mathrm{C} 9 \mathrm{H} 20 \mathrm{O} 2 \mathrm{~N}$ & -0.28 & {$[\mathrm{M}+\mathrm{H}]^{+}$} & I \\
\hline 175.1520 & I & I & I & I \\
\hline 176.1642 & $\mathrm{C} 9 \mathrm{H} 22 \mathrm{O} 2 \mathrm{~N}$ & -0.28 & {$[\mathrm{M}+\mathrm{H}]^{+}$} & / \\
\hline 178.1435 & $\mathrm{C} 8 \mathrm{H} 20 \mathrm{O} 3 \mathrm{~N}$ & -0.33 & {$[\mathrm{M}+\mathrm{H}]^{+}$} & I \\
\hline 179.1468 & $\mathrm{C} 2 \mathrm{H} 15 \mathrm{~N} 10$ & -0.76 & {$[\mathrm{M}+\mathrm{H}]^{+}$} & / \\
\hline 180.1016 & $\mathrm{C} 10 \mathrm{H} 14 \mathrm{O} 2 \mathrm{~N}$ & -0.36 & {$[\mathrm{M}+\mathrm{H}]^{+}$} & I \\
\hline 181.0854 & $\mathrm{C} 10 \mathrm{H} 13 \mathrm{O} 3$ & -0.4 & {$[\mathrm{M}+\mathrm{H}]^{+}$} & I \\
\hline 181.1050 & $\mathrm{C} 3 \mathrm{H} 13 \mathrm{O} 3 \mathrm{~N} 6$ & 0.52 & {$[\mathrm{M}+\mathrm{H}]^{+}$} & / \\
\hline 183.1012 & $\mathrm{C} 10 \mathrm{H} 15 \mathrm{O} 3$ & -0.32 & {$[\mathrm{M}+\mathrm{H}]^{+}$} & I \\
\hline 183.1377 & $\mathrm{C} 11 \mathrm{H} 19 \mathrm{O} 2$ & -0.31 & {$[\mathrm{M}+\mathrm{H}]^{+}$} & / \\
\hline 183.1740 & $\mathrm{C} 12 \mathrm{H} 23 \mathrm{O}$ & -0.29 & {$[\mathrm{M}+\mathrm{H}]^{+}$} & I \\
\hline 184.1329 & $\mathrm{C} 10 \mathrm{H} 18 \mathrm{O} 2 \mathrm{~N}$ & -0.31 & {$[\mathrm{M}+\mathrm{H}]^{+}$} & / \\
\hline 185.1533 & $\mathrm{C} 11 \mathrm{H} 21 \mathrm{O} 2$ & -0.34 & {$[\mathrm{M}+\mathrm{H}]^{+}$} & 5-heptyldihydro-2(3H)-furanone \\
\hline 188.1641 & $\mathrm{C} 10 \mathrm{H} 22 \mathrm{O} 2 \mathrm{~N}$ & -0.37 & {$[\mathrm{M}+\mathrm{H}]^{+}$} & 1 \\
\hline 195.1014 & $\mathrm{C} 11 \mathrm{H} 15 \mathrm{O} 3$ & -0.33 & {$[\mathrm{M}+\mathrm{H}]^{+}$} & l \\
\hline 196.1691 & $\mathrm{C} 12 \mathrm{H} 22 \mathrm{ON}$ & -0.36 & {$[\mathrm{M}+\mathrm{H}]^{+}$} & / \\
\hline 197.1532 & $\mathrm{C} 12 \mathrm{H} 21 \mathrm{O} 2$ & -0.39 & {$[\mathrm{M}+\mathrm{H}]^{+}$} & l \\
\hline 198.1121 & $\mathrm{C} 10 \mathrm{H} 16 \mathrm{O} 3 \mathrm{~N}$ & -0.39 & {$[\mathrm{M}+\mathrm{H}]^{+}$} & / \\
\hline 198.1484 & $\mathrm{C} 11 \mathrm{H} 20 \mathrm{O} 2 \mathrm{~N}$ & -0.39 & {$[\mathrm{M}+\mathrm{H}]^{+}$} & I \\
\hline 198.1848 & $\mathrm{C} 12 \mathrm{H} 24 \mathrm{ON}$ & -0.41 & {$[\mathrm{M}+\mathrm{H}]^{+}$} & l \\
\hline 199.1883 & I & / & I & / \\
\hline 200.1641 & $\mathrm{C} 11 \mathrm{H} 22 \mathrm{O} 2 \mathrm{~N}$ & -0.4 & {$[\mathrm{M}+\mathrm{H}]^{+}$} & I \\
\hline 202.1798 & $\mathrm{C} 11 \mathrm{H} 24 \mathrm{O} 2 \mathrm{~N}$ & -0.4 & {$[\mathrm{M}+\mathrm{H}]^{+}$} & / \\
\hline 203.1467 & C4H15N10 & -0.8 & {$[\mathrm{M}+\mathrm{H}]^{+}$} & I \\
\hline 206.1747 & $\mathrm{C} 10 \mathrm{H} 24 \mathrm{O} 3 \mathrm{~N}$ & -0.43 & {$[\mathrm{M}+\mathrm{H}]^{+}$} & I \\
\hline 211.1689 & $\mathrm{C} 13 \mathrm{H} 23 \mathrm{O} 2$ & -0.46 & {$[\mathrm{M}+\mathrm{H}]^{+}$} & / \\
\hline 212.1276 & $\mathrm{C} 11 \mathrm{H} 18 \mathrm{O} 3 \mathrm{~N}$ & -0.47 & {$[\mathrm{M}+\mathrm{H}]^{+}$} & I \\
\hline 212.1640 & $\mathrm{C} 12 \mathrm{H} 22 \mathrm{O} 2 \mathrm{~N}$ & -0.47 & {$[\mathrm{M}+\mathrm{H}]^{+}$} & I \\
\hline 212.1721 & 1 & I & I & I \\
\hline 213.1379 & $\mathrm{C} 14 \mathrm{H} 17 \mathrm{~N} 2$ & -0.45 & {$[\mathrm{M}+\mathrm{H}]^{+}$} & I \\
\hline 213.1845 & $\mathrm{C} 13 \mathrm{H} 25 \mathrm{O} 2$ & -0.47 & {$[\mathrm{M}+\mathrm{H}]^{+}$} & / \\
\hline 214.1433 & $\mathrm{C} 11 \mathrm{H} 20 \mathrm{O} 3 \mathrm{~N}$ & -0.49 & {$[\mathrm{M}+\mathrm{H}]^{+}$} & I \\
\hline 214.1797 & $\mathrm{C} 12 \mathrm{H} 24 \mathrm{O} 2 \mathrm{~N}$ & -0.47 & {$[\mathrm{M}+\mathrm{H}]^{+}$} & I \\
\hline 214.1879 & 1 & I & I & I \\
\hline 215.1387 & $\mathrm{C} 10 \mathrm{H} 19 \mathrm{O} 3 \mathrm{~N} 2$ & -0.38 & {$[\mathrm{M}+\mathrm{H}]^{+}$} & I \\
\hline 216.1955 & $\mathrm{C} 12 \mathrm{H} 26 \mathrm{O} 2 \mathrm{~N}$ & -0.46 & {$[\mathrm{M}+\mathrm{H}]^{+}$} & I \\
\hline
\end{tabular}




\begin{tabular}{|c|c|c|c|c|}
\hline 217.1001 & l & / & I & I \\
\hline 220.1902 & $\mathrm{C} 11 \mathrm{H} 26 \mathrm{O} 3 \mathrm{~N}$ & -0.5 & {$[\mathrm{M}+\mathrm{H}]^{+}$} & I \\
\hline 223.1686 & $\mathrm{C} 14 \mathrm{H} 23 \mathrm{O} 2$ & -0.52 & {$[\mathrm{M}+\mathrm{H}]^{+}$} & I \\
\hline 226.1432 & $\mathrm{C} 12 \mathrm{H} 20 \mathrm{O} 3 \mathrm{~N}$ & -0.53 & {$[\mathrm{M}+\mathrm{H}]^{+}$} & / \\
\hline 227.1997 & $\mathrm{C} 14 \mathrm{H} 27 \mathrm{O} 2$ & -0.57 & {$[\mathrm{M}+\mathrm{H}]^{+}$} & 5-decyldihydro-2(3H)-furanone \\
\hline 228.1590 & $\mathrm{C} 12 \mathrm{H} 22 \mathrm{O} 3 \mathrm{~N}$ & -0.43 & {$[\mathrm{M}+\mathrm{H}]^{+}$} & 1 \\
\hline 228.1954 & $\mathrm{C} 13 \mathrm{H} 26 \mathrm{O} 2 \mathrm{~N}$ & -0.46 & {$[\mathrm{M}+\mathrm{H}]^{+}$} & / \\
\hline 229.1623 & C6H17N10 & -0.88 & {$[\mathrm{M}+\mathrm{H}]^{+}$} & I \\
\hline 230.1746 & $\mathrm{C} 12 \mathrm{H} 24 \mathrm{O} 3 \mathrm{~N}$ & -0.46 & {$[\mathrm{M}+\mathrm{H}]^{+}$} & / \\
\hline 230.2107 & $\mathrm{C} 13 \mathrm{H} 28 \mathrm{O} 2 \mathrm{~N}$ & -0.48 & {$[\mathrm{M}+\mathrm{H}]^{+}$} & I \\
\hline 231.1779 & C6H19N10 & -0.94 & {$[\mathrm{M}+\mathrm{H}]^{+}$} & / \\
\hline 237.1116 & $\mathrm{C} 13 \mathrm{H} 17 \mathrm{O} 4$ & -0.52 & {$[\mathrm{M}+\mathrm{H}]^{+}$} & / \\
\hline 240.1588 & $\mathrm{C} 13 \mathrm{H} 22 \mathrm{O} 3 \mathrm{~N}$ & -0.57 & {$[\mathrm{M}+\mathrm{H}]^{+}$} & I \\
\hline 240.1953 & $\mathrm{C} 14 \mathrm{H} 26 \mathrm{O} 2 \mathrm{~N}$ & -0.56 & {$[\mathrm{M}+\mathrm{H}]^{+}$} & / \\
\hline 242.1745 & $\mathrm{C} 13 \mathrm{H} 24 \mathrm{O} 3 \mathrm{~N}$ & -0.61 & {$[\mathrm{M}+\mathrm{H}]^{+}$} & I \\
\hline 242.2110 & $\mathrm{C} 14 \mathrm{H} 28 \mathrm{O} 2 \mathrm{~N}$ & -0.65 & {$[\mathrm{M}+\mathrm{H}]^{+}$} & I \\
\hline 243.1697 & $\mathrm{C} 12 \mathrm{H} 23 \mathrm{O} 3 \mathrm{~N} 2$ & -0.53 & {$[\mathrm{M}+\mathrm{H}]^{+}$} & I \\
\hline 243.1779 & C7H19N10 & -0.92 & {$[\mathrm{M}+\mathrm{H}]^{+}$} & I \\
\hline 244.1902 & $\mathrm{C} 13 \mathrm{H} 26 \mathrm{O} 3 \mathrm{~N}$ & -0.49 & {$[\mathrm{M}+\mathrm{H}]^{+}$} & I \\
\hline 253.1540 & $\mathrm{C} 13 \mathrm{H} 21 \mathrm{O} 3 \mathrm{~N} 2$ & -0.63 & {$[\mathrm{M}+\mathrm{H}]^{+}$} & I \\
\hline 254.1381 & $\mathrm{C} 13 \mathrm{H} 20 \mathrm{O} 4 \mathrm{~N}$ & -0.64 & {$[\mathrm{M}+\mathrm{H}]^{+}$} & I \\
\hline 254.1745 & $\mathrm{C} 14 \mathrm{H} 24 \mathrm{O} 3 \mathrm{~N}$ & -0.58 & {$[\mathrm{M}+\mathrm{H}]^{+}$} & / \\
\hline 255.1414 & C6H19O5N6 & 0.26 & {$[\mathrm{M}+\mathrm{H}]^{+}$} & I \\
\hline 256.1538 & $\mathrm{C} 13 \mathrm{H} 22 \mathrm{O} 4 \mathrm{~N}$ & -0.62 & {$[\mathrm{M}+\mathrm{H}]^{+}$} & l \\
\hline 256.1901 & $\mathrm{C} 14 \mathrm{H} 26 \mathrm{O} 3 \mathrm{~N}$ & -0.65 & {$[\mathrm{M}+\mathrm{H}]^{+}$} & / \\
\hline 258.2058 & $\mathrm{C} 14 \mathrm{H} 28 \mathrm{O} 3 \mathrm{~N}$ & -0.58 & {$[\mathrm{M}+\mathrm{H}]^{+}$} & I \\
\hline 268.1537 & $\mathrm{C} 14 \mathrm{H} 22 \mathrm{O} 4 \mathrm{~N}$ & -0.57 & {$[\mathrm{M}+\mathrm{H}]^{+}$} & / \\
\hline 270.1328 & $\mathrm{C} 13 \mathrm{H} 20 \mathrm{O} 5 \mathrm{~N}$ & -0.63 & {$[\mathrm{M}+\mathrm{H}]^{+}$} & I \\
\hline 272.2212 & $\mathrm{C} 15 \mathrm{H} 30 \mathrm{O} 3 \mathrm{~N}$ & -0.74 & {$[\mathrm{M}+\mathrm{H}]^{+}$} & I \\
\hline 284.2214 & $\mathrm{C} 16 \mathrm{H} 30 \mathrm{O} 3 \mathrm{~N}$ & -0.68 & {$[\mathrm{M}+\mathrm{H}]^{+}$} & / \\
\hline 286.2005 & $\mathrm{C} 15 \mathrm{H} 28 \mathrm{O} 4 \mathrm{~N}$ & -0.75 & {$[\mathrm{M}+\mathrm{H}]^{+}$} & I \\
\hline 286.2369 & $\mathrm{C} 16 \mathrm{H} 32 \mathrm{O} 3 \mathrm{~N}$ & -0.73 & {$[\mathrm{M}+\mathrm{H}]^{+}$} & I \\
\hline 298.2734 & $\mathrm{C} 18 \mathrm{H} 36 \mathrm{O} 2 \mathrm{~N}$ & -0.71 & {$[\mathrm{M}+\mathrm{H}]^{+}$} & / \\
\hline 300.2525 & $\mathrm{C} 17 \mathrm{H} 34 \mathrm{O} 3 \mathrm{~N}$ & -0.7 & {$[\mathrm{M}+\mathrm{H}]^{+}$} & I \\
\hline 310.1642 & $\mathrm{C} 16 \mathrm{H} 24 \mathrm{O} 5 \mathrm{~N}$ & -0.81 & {$[\mathrm{M}+\mathrm{H}]^{+}$} & / \\
\hline 71.0125 & $\mathrm{C} 3 \mathrm{H} 3 \mathrm{O} 2$ & -1.4 & {$[\mathrm{M}-\mathrm{H}]^{-}$} & I \\
\hline 72.9917 & $\mathrm{C} 2 \mathrm{HO} 3$ & -1.39 & {$[\mathrm{M}-\mathrm{H}]^{-}$} & l \\
\hline 83.0124 & $\mathrm{C} 4 \mathrm{H} 3 \mathrm{O} 2$ & -1.4 & {$[\mathrm{M}-\mathrm{H}]^{-}$} & / \\
\hline 97.0281 & I & I & I & I \\
\hline 99.0074 & $\mathrm{C} 4 \mathrm{H} 3 \mathrm{O} 3$ & -1.37 & {$[\mathrm{M}-\mathrm{H}]^{-}$} & I \\
\hline 100.9866 & $\mathrm{C} 3 \mathrm{HO} 4$ & -1.37 & {$[\mathrm{M}-\mathrm{H}]^{-}$} & I \\
\hline 101.0231 & I & I & I & I \\
\hline 103.0023 & $\mathrm{C} 3 \mathrm{H} 3 \mathrm{O} 4$ & -1.36 & {$[\mathrm{M}-\mathrm{H}]^{-}$} & I \\
\hline 105.0180 & C3H5O4 & -1.36 & {$[\mathrm{M}-\mathrm{H}]^{-}$} & I \\
\hline
\end{tabular}




\begin{tabular}{|c|c|c|c|c|}
\hline 113.0231 & CHON6 & 1.31 & {$[\mathrm{M}-\mathrm{H}]^{-}$} & l \\
\hline 115.0024 & $\mathrm{C} 4 \mathrm{H} 3 \mathrm{O} 4$ & -1.31 & {$[\mathrm{M}-\mathrm{H}]^{-}$} & l \\
\hline 115.0388 & $\mathrm{C} 5 \mathrm{H} 7 \mathrm{O} 3$ & -1.32 & {$[\mathrm{M}-\mathrm{H}]^{-}$} & l \\
\hline 117.0180 & $\mathrm{C} 4 \mathrm{H} 5 \mathrm{O} 4$ & -1.32 & {$[\mathrm{M}-\mathrm{H}]^{-}$} & I \\
\hline 117.0543 & C5H9O3 & -1.33 & {$[\mathrm{M}-\mathrm{H}]^{-}$} & l \\
\hline 125.0231 & C6H5O3 & -1.3 & {$[\mathrm{M}-\mathrm{H}]^{-}$} & I \\
\hline 127.0388 & $\mathrm{C} 6 \mathrm{H} 7 \mathrm{O} 3$ & -1.27 & {$[\mathrm{M}-\mathrm{H}]^{-}$} & l \\
\hline 129.0180 & $\mathrm{C} 5 \mathrm{H} 5 \mathrm{O} 4$ & -1.26 & {$[\mathrm{M}-\mathrm{H}]^{-}$} & l \\
\hline 129.0545 & C6H9O3 & -1.26 & {$[\mathrm{M}-\mathrm{H}]^{-}$} & l \\
\hline 130.0578 & I & I & I & l \\
\hline 133.0130 & C4H5O5 & -1.26 & {$[\mathrm{M}-\mathrm{H}]^{-}$} & l \\
\hline 139.0752 & $\mathrm{C} 8 \mathrm{H} 11 \mathrm{O} 2$ & -1.24 & {$[\mathrm{M}-\mathrm{H}]^{-}$} & l \\
\hline 141.0545 & C7H9O3 & -1.21 & {$[\mathrm{M}-\mathrm{H}]^{-}$} & l \\
\hline 143.0702 & C7H11O3 & -1.17 & {$[\mathrm{M}-\mathrm{H}]^{-}$} & l \\
\hline 144.0735 & I & I & I & l \\
\hline 145.0494 & C6H9O4 & -1.19 & {$[\mathrm{M}-\mathrm{H}]^{-}$} & l \\
\hline 145.0859 & $\mathrm{C} 7 \mathrm{H} 13 \mathrm{O} 3$ & -1.19 & {$[\mathrm{M}-\mathrm{H}]^{-}$} & I \\
\hline 147.0287 & C5H7O5 & -1.17 & {$[\mathrm{M}-\mathrm{H}]^{-}$} & l \\
\hline 153.0909 & $\mathrm{C} 9 \mathrm{H} 13 \mathrm{O} 2$ & -1.15 & {$[\mathrm{M}-\mathrm{H}]^{-}$} & l \\
\hline 155.0702 & $\mathrm{C} 8 \mathrm{H} 11 \mathrm{O} 3$ & -1.14 & {$[\mathrm{M}-\mathrm{H}]^{-}$} & l \\
\hline 155.1066 & $\mathrm{C} 9 \mathrm{H} 15 \mathrm{O} 2$ & -1.13 & {$[\mathrm{M}-\mathrm{H}]^{-}$} & Nonenoic acid \\
\hline 156.1100 & I & I & I & I \\
\hline 157.0495 & C7H9O4 & -1.11 & {$[\mathrm{M}-\mathrm{H}]^{-}$} & l \\
\hline 159.0652 & C7H11O4 & -1.11 & {$[\mathrm{M}-\mathrm{H}]^{-}$} & l \\
\hline 161.0444 & C6H9O5 & -1.13 & {$[\mathrm{M}-\mathrm{H}]^{-}$} & l \\
\hline 165.0546 & С9H9O3 & -1.1 & {$[\mathrm{M}-\mathrm{H}]^{-}$} & l \\
\hline 167.1067 & $\mathrm{C} 10 \mathrm{H} 15 \mathrm{O} 2$ & -1.03 & {$[\mathrm{M}-\mathrm{H}]^{-}$} & l \\
\hline 168.1101 & C3H14O3N5 & -0.12 & {$[\mathrm{M}-\mathrm{H}]^{-}$} & l \\
\hline 169.1224 & $\mathrm{C} 10 \mathrm{H} 17 \mathrm{O} 2$ & -1.01 & {$[\mathrm{M}-\mathrm{H}]^{-}$} & Decenoic acid \\
\hline 170.1256 & I & I & I & I \\
\hline 175.0601 & C7H11O5 & -1.04 & {$[\mathrm{M}-\mathrm{H}]^{-}$} & l \\
\hline 181.0860 & $\mathrm{C} 10 \mathrm{H} 13 \mathrm{O} 3$ & -1.01 & {$[\mathrm{M}-\mathrm{H}]^{-}$} & l \\
\hline 183.1018 & $\mathrm{C} 10 \mathrm{H} 15 \mathrm{O} 3$ & -0.95 & {$[\mathrm{M}-\mathrm{H}]^{-}$} & l \\
\hline 184.1051 & I & I & I & l \\
\hline 190.0712 & $\mathrm{C} 7 \mathrm{H} 12 \mathrm{O} 5 \mathrm{~N}$ & -0.89 & {$[\mathrm{M}-\mathrm{H}]^{-}$} & l \\
\hline 196.0970 & $\mathrm{C} 10 \mathrm{H} 14 \mathrm{O} 3 \mathrm{~N}$ & -0.89 & {$[\mathrm{M}-\mathrm{H}]^{-}$} & l \\
\hline 197.0811 & $\mathrm{C} 10 \mathrm{H} 13 \mathrm{O} 4$ & -0.87 & {$[\mathrm{M}-\mathrm{H}]^{-}$} & l \\
\hline 199.0967 & $\mathrm{C} 10 \mathrm{H} 15 \mathrm{O} 4$ & -0.88 & {$[\mathrm{M}-\mathrm{H}]^{-}$} & l \\
\hline 201.1124 & $\mathrm{C} 10 \mathrm{H} 17 \mathrm{O} 4$ & -0.85 & {$[\mathrm{M}-\mathrm{H}]^{-}$} & l \\
\hline 202.1075 & C9H16O4N & -0.84 & {$[\mathrm{M}-\mathrm{H}]^{-}$} & l \\
\hline 206.1026 & $\mathrm{C} 8 \mathrm{H} 16 \mathrm{O} 5 \mathrm{~N}$ & -0.81 & {$[\mathrm{M}-\mathrm{H}]^{-}$} & l \\
\hline 211.1332 & $\mathrm{C} 12 \mathrm{H} 19 \mathrm{O} 3$ & -0.75 & {$[\mathrm{M}-\mathrm{H}]^{-}$} & l \\
\hline 212.1366 & C5H18O4N5 & 0.11 & {$[\mathrm{M}-\mathrm{H}]^{-}$} & l \\
\hline 213.0761 & $\mathrm{C} 10 \mathrm{H} 13 \mathrm{O} 5$ & -0.77 & {$[\mathrm{M}-\mathrm{H}]^{-}$} & I \\
\hline
\end{tabular}




\begin{tabular}{|c|c|c|c|c|}
\hline 214.1078 & $\mathrm{C} 10 \mathrm{H} 16 \mathrm{O} 4 \mathrm{~N}$ & -0.77 & {$[\mathrm{M}-\mathrm{H}]^{-}$} & l \\
\hline 215.0917 & $\mathrm{C} 10 \mathrm{H} 15 \mathrm{O} 5$ & -0.77 & {$[\mathrm{M}-\mathrm{H}]^{-}$} & I \\
\hline 216.1234 & C10H18O4N & -0.76 & {$[\mathrm{M}-\mathrm{H}]^{-}$} & I \\
\hline 217.1074 & $\mathrm{C} 10 \mathrm{H} 17 \mathrm{O} 5$ & -0.73 & {$[\mathrm{M}-\mathrm{H}]^{-}$} & I \\
\hline 230.1028 & $\mathrm{C} 10 \mathrm{H} 16 \mathrm{O} 5 \mathrm{~N}$ & -0.67 & {$[\mathrm{M}-\mathrm{H}]^{-}$} & I \\
\hline 231.0867 & $\mathrm{C} 10 \mathrm{H} 15 \mathrm{O} 6$ & -0.64 & {$[\mathrm{M}-\mathrm{H}]^{-}$} & I \\
\hline 232.0819 & $\mathrm{C} 9 \mathrm{H} 14 \mathrm{O} 6 \mathrm{~N}$ & -0.63 & {$[\mathrm{M}-\mathrm{H}]^{-}$} & I \\
\hline 232.1184 & $\mathrm{C} 10 \mathrm{H} 18 \mathrm{O} 5 \mathrm{~N}$ & -0.64 & {$[\mathrm{M}-\mathrm{H}]^{-}$} & I \\
\hline 233.1024 & $\mathrm{C} 10 \mathrm{H} 17 \mathrm{O} 6$ & -0.64 & {$[\mathrm{M}-\mathrm{H}]^{-}$} & I \\
\hline 246.0978 & $\mathrm{C} 10 \mathrm{H} 16 \mathrm{O} 6 \mathrm{~N}$ & -0.54 & {$[\mathrm{M}-\mathrm{H}]^{-}$} & I \\
\hline 248.0772 & $\mathrm{C} 9 \mathrm{H} 14 \mathrm{O} 7 \mathrm{~N}$ & -0.48 & {$[\mathrm{M}-\mathrm{H}]^{-}$} & / \\
\hline 248.1134 & $\mathrm{C} 10 \mathrm{H} 18 \mathrm{O} 6 \mathrm{~N}$ & -0.51 & {$[\mathrm{M}-\mathrm{H}]^{-}$} & I \\
\hline 258.1343 & $\mathrm{C} 12 \mathrm{H} 20 \mathrm{O} 5 \mathrm{~N}$ & -0.41 & {$[\mathrm{M}-\mathrm{H}]^{-}$} & I \\
\hline 262.0928 & $\mathrm{C} 10 \mathrm{H} 16 \mathrm{O} 7 \mathrm{~N}$ & -0.37 & {$[\mathrm{M}-\mathrm{H}]^{-}$} & I \\
\hline 264.1084 & $\mathrm{C} 10 \mathrm{H} 18 \mathrm{O} 7 \mathrm{~N}$ & -0.38 & {$[\mathrm{M}-\mathrm{H}]^{-}$} & I \\
\hline 266.0878 & C9H16O8N & -0.36 & {$[\mathrm{M}-\mathrm{H}]^{-}$} & l \\
\hline 274.1293 & $\mathrm{C} 12 \mathrm{H} 20 \mathrm{O} 6 \mathrm{~N}$ & -0.31 & {$[\mathrm{M}-\mathrm{H}]^{-}$} & I \\
\hline 278.0877 & C10H16O8N & -0.39 & {$[\mathrm{M}-\mathrm{H}]^{-}$} & I \\
\hline 279.2326 & $\mathrm{C} 18 \mathrm{H} 31 \mathrm{O} 2$ & -0.35 & {$[\mathrm{M}-\mathrm{H}]^{-}$} & I \\
\hline 280.1035 & $\mathrm{C} 10 \mathrm{H} 18 \mathrm{O} 8 \mathrm{~N}$ & -0.42 & {$[\mathrm{M}-\mathrm{H}]^{-}$} & l \\
\hline 280.2359 & $\mathrm{C} 11 \mathrm{H} 30 \mathrm{O} 3 \mathrm{~N} 5$ & 0.53 & {$[\mathrm{M}-\mathrm{H}]^{-}$} & I \\
\hline 293.0988 & $\mathrm{C} 10 \mathrm{H} 17 \mathrm{O} 8 \mathrm{~N} 2$ & -0.31 & {$[\mathrm{M}-\mathrm{H}]^{-}$} & I \\
\hline 301.2380 & C17H33O4 & -0.45 & {$[\mathrm{M}-\mathrm{H}]^{-}$} & I \\
\hline 325.2379 & $\mathrm{C} 19 \mathrm{H} 33 \mathrm{O} 4$ & -0.5 & {$[\mathrm{M}-\mathrm{H}]^{-}$} & I \\
\hline 326.2333 & C18H32O4N & -0.47 & {$[\mathrm{M}-\mathrm{H}]^{-}$} & I \\
\hline 327.2365 & C11H31O5N6 & 0.4 & {$[\mathrm{M}-\mathrm{H}]^{-}$} & I \\
\hline 327.2535 & C19H35O4 & -0.49 & {$[\mathrm{M}-\mathrm{H}]^{-}$} & I \\
\hline 328.2489 & C18H34O4N & -0.46 & {$[\mathrm{M}-\mathrm{H}]^{-}$} & I \\
\hline 329.2523 & C12H29ON10 & -0.92 & {$[\mathrm{M}-\mathrm{H}]^{-}$} & I \\
\hline 342.2281 & $\mathrm{C} 18 \mathrm{H} 32 \mathrm{O} 5 \mathrm{~N}$ & -0.55 & {$[\mathrm{M}-\mathrm{H}]^{-}$} & I \\
\hline 344.2438 & $\mathrm{C} 18 \mathrm{H} 34 \mathrm{O} 5 \mathrm{~N}$ & -0.55 & {$[\mathrm{M}-\mathrm{H}]^{-}$} & l \\
\hline 451.3422 & $\mathrm{C} 27 \mathrm{H} 47 \mathrm{O} 5$ & -0.68 & {$[\mathrm{M}-\mathrm{H}]^{-}$} & I \\
\hline
\end{tabular}

Table S5. Detailed information of ions classified in Group 4 in Figure 1. ${ }^{4-12}$

\begin{tabular}{ccccc}
\hline $\boldsymbol{m} / \boldsymbol{z}$ & Composition & $\boldsymbol{\Delta} \mathbf{m m u}$ & Ionmode & Compounds \\
\hline 139.0751 & $\mathrm{C} 8 \mathrm{H} 11 \mathrm{O} 2$ & -0.22 & {$[\mathrm{M}+\mathrm{H}]^{+}$} & $/$ \\
155.0700 & $\mathrm{C} 8 \mathrm{H} 11 \mathrm{O} 3$ & -0.29 & {$[\mathrm{M}+\mathrm{H}]^{+}$} & $/$ \\
160.0965 & $\mathrm{C} 7 \mathrm{H} 14 \mathrm{O} 3 \mathrm{~N}$ & -0.32 & {$[\mathrm{M}+\mathrm{H}]^{+}$} & $/$ \\
174.1122 & $\mathrm{C} 8 \mathrm{H} 16 \mathrm{O} 3 \mathrm{~N}$ & -0.27 & {$[\mathrm{M}+\mathrm{H}]^{+}$} & $/$ \\
175.1155 & $/$ & $/$ & $/$ & $/$ \\
184.1693 & $\mathrm{C} 11 \mathrm{H} 22 \mathrm{ON}$ & -0.36 & {$[\mathrm{M}+\mathrm{H}]^{+}$} & $/$ \\
\hline
\end{tabular}




\begin{tabular}{|c|c|c|c|c|}
\hline 216.1226 & $\mathrm{C} 10 \mathrm{H} 18 \mathrm{O} 4 \mathrm{~N}$ & -0.42 & {$[\mathrm{M}+\mathrm{H}]^{+}$} & I \\
\hline 230.1382 & C11H20O4N & -0.48 & {$[\mathrm{M}+\mathrm{H}]^{+}$} & / \\
\hline 244.1538 & $\mathrm{C} 12 \mathrm{H} 22 \mathrm{O} 4 \mathrm{~N}$ & -0.52 & {$[\mathrm{M}+\mathrm{H}]^{+}$} & I \\
\hline 260.1850 & $\mathrm{C} 13 \mathrm{H} 26 \mathrm{O} 4 \mathrm{~N}$ & -0.63 & {$[\mathrm{M}+\mathrm{H}]^{+}$} & / \\
\hline 269.2106 & C16H29O3 & -0.65 & {$[\mathrm{M}+\mathrm{H}]^{+}$} & I \\
\hline 272.1849 & $\mathrm{C} 14 \mathrm{H} 26 \mathrm{O} 4 \mathrm{~N}$ & -0.72 & {$[\mathrm{M}+\mathrm{H}]^{+}$} & / \\
\hline 272.2576 & $\mathrm{C} 16 \mathrm{H} 34 \mathrm{O} 2 \mathrm{~N}$ & -0.75 & {$[\mathrm{M}+\mathrm{H}]^{+}$} & I \\
\hline 328.2838 & C19H38O3N & -0.89 & {$[\mathrm{M}+\mathrm{H}]^{+}$} & / \\
\hline 77.9945 & I & I & I & I \\
\hline 79.0023 & I & I & I & / \\
\hline 85.0645 & $\mathrm{C} 5 \mathrm{H} 9 \mathrm{O}$ & -1.4 & {$[\mathrm{M}-\mathrm{H}]^{-}$} & / \\
\hline 85.9995 & I & I & I & I \\
\hline 99.0438 & $\mathrm{C} 5 \mathrm{H} 7 \mathrm{O} 2$ & 1.36 & {$[\mathrm{M}-\mathrm{H}]^{-}$} & / \\
\hline 99.0801 & C6H11O & -1.37 & {$[\mathrm{M}-\mathrm{H}]^{-}$} & / \\
\hline 101.0595 & $\mathrm{C} 5 \mathrm{H} 9 \mathrm{O} 2$ & 1.37 & {$[\mathrm{M}-\mathrm{H}]^{-}$} & / \\
\hline 113.0594 & C6H9O2 & 1.34 & {$[\mathrm{M}-\mathrm{H}]^{-}$} & Hexenoic acid \\
\hline 115.0751 & C6H11O2 & -1.33 & {$[\mathrm{M}-\mathrm{H}]^{-}$} & Hexanoic acid \\
\hline 116.0785 & I & I & I & l \\
\hline 124.0154 & $\mathrm{C} 4 \mathrm{H} 2 \mathrm{O} 2 \mathrm{~N} 3$ & 0.06 & {$[\mathrm{M}-\mathrm{H}]^{-}$} & / \\
\hline 125.0594 & C7H9O2 & -1.31 & {$[\mathrm{M}-\mathrm{H}]^{-}$} & I \\
\hline 125.0959 & C8H13O & -1.3 & {$[\mathrm{M}-\mathrm{H}]^{-}$} & I \\
\hline 127.0752 & C7H11O2 & -1.27 & {$[\mathrm{M}-\mathrm{H}]^{-}$} & Heptenoic acid \\
\hline 127.1116 & $\mathrm{C} 8 \mathrm{H} 15 \mathrm{O}$ & -1.27 & {$[\mathrm{M}-\mathrm{H}]^{-}$} & I \\
\hline 129.0909 & $\mathrm{C} 7 \mathrm{H} 13 \mathrm{O} 2$ & -1.26 & {$[\mathrm{M}-\mathrm{H}]^{-}$} & Heptanoic acid \\
\hline 131.0701 & C6H11O3 & -1.25 & {$[\mathrm{M}-\mathrm{H}]^{-}$} & 1 \\
\hline 139.1118 & С9H15O & -1.2 & {$[\mathrm{M}-\mathrm{H}]^{-}$} & I \\
\hline 142.0944 & I & I & I & I \\
\hline 144.1100 & C6H14ON3 & -4.3 & {$[\mathrm{M}-\mathrm{H}]^{-}$} & / \\
\hline 146.0572 & $\mathrm{C} 4 \mathrm{H} 8 \mathrm{O} 3 \mathrm{~N} 3$ & 0.16 & {$[\mathrm{M}-\mathrm{H}]^{-}$} & / \\
\hline 147.0651 & C6H11O4 & -1.18 & {$[\mathrm{M}-\mathrm{H}]^{-}$} & I \\
\hline 148.0730 & C4H10O3N3 & 0.17 & {$[\mathrm{M}-\mathrm{H}]^{-}$} & I \\
\hline 150.0008 & I & I & I & / \\
\hline 153.0545 & C8H9O3 & -1.16 & {$[\mathrm{M}-\mathrm{H}]^{-}$} & / \\
\hline 154.0988 & C7H12ON3 & 0.19 & {$[\mathrm{M}-\mathrm{H}]^{-}$} & / \\
\hline 155.0338 & C7H7O4 & -1.17 & {$[\mathrm{M}-\mathrm{H}]^{-}$} & / \\
\hline 161.0810 & C7H13O4 & -1.07 & {$[\mathrm{M}-\mathrm{H}]^{-}$} & / \\
\hline 169.0497 & C8H9O4 & -1.04 & {$[\mathrm{M}-\mathrm{H}]^{-}$} & / \\
\hline 169.0860 & C9H13O3 & -0.99 & {$[\mathrm{M}-\mathrm{H}]^{-}$} & / \\
\hline 170.0893 & $\mathrm{C} 2 \mathrm{H} 12 \mathrm{O} 4 \mathrm{~N} 5$ & -0.12 & {$[\mathrm{M}-\mathrm{H}]^{-}$} & / \\
\hline 173.0445 & C7H9O5 & -1.06 & {$[\mathrm{M}-\mathrm{H}]^{-}$} & / \\
\hline 175.0237 & C6H7O6 & -1.04 & {$[\mathrm{M}-\mathrm{H}]^{-}$} & / \\
\hline 182.0939 & $\mathrm{C} 8 \mathrm{H} 12 \mathrm{O} 2 \mathrm{~N} 3$ & 0.38 & {$[\mathrm{M}-\mathrm{H}]^{-}$} & / \\
\hline 183.0652 & C9H11O4 & -1 & {$[\mathrm{M}-\mathrm{H}]^{-}$} & I \\
\hline 183.1381 & $\mathrm{C} 11 \mathrm{H} 19 \mathrm{O} 2$ & -0.99 & {$[\mathrm{M}-\mathrm{H}]^{-}$} & Undecylenic acid \\
\hline
\end{tabular}




\begin{tabular}{|c|c|c|c|c|}
\hline 184.1094 & $\mathrm{C} 8 \mathrm{H} 14 \mathrm{O} 2 \mathrm{~N} 3$ & 0.35 & {$[\mathrm{M}-\mathrm{H}]^{-}$} & I \\
\hline 185.1174 & $\mathrm{C} 10 \mathrm{H} 17 \mathrm{O} 3$ & -0.97 & {$[\mathrm{M}-\mathrm{H}]^{-}$} & / \\
\hline 187.0604 & C8H11O5 & -0.93 & {$[\mathrm{M}-\mathrm{H}]^{-}$} & I \\
\hline 202.1202 & C8H16O3N3 & 0.5 & {$[\mathrm{M}-\mathrm{H}]^{-}$} & / \\
\hline 204.0870 & $\mathrm{C} 8 \mathrm{H} 14 \mathrm{O} 5 \mathrm{~N}$ & -0.81 & {$[\mathrm{M}-\mathrm{H}]^{-}$} & / \\
\hline 205.1072 & С9H17O5 & -0.86 & {$[\mathrm{M}-\mathrm{H}]^{-}$} & I \\
\hline 209.1176 & $\mathrm{C} 12 \mathrm{H} 17 \mathrm{O} 3$ & -0.74 & {$[\mathrm{M}-\mathrm{H}]^{-}$} & / \\
\hline 211.0967 & $\mathrm{C} 11 \mathrm{H} 15 \mathrm{O} 4$ & -0.75 & {$[\mathrm{M}-\mathrm{H}]^{-}$} & / \\
\hline 213.1124 & $\mathrm{C} 11 \mathrm{H} 17 \mathrm{O} 4$ & -0.78 & {$[\mathrm{M}-\mathrm{H}]^{-}$} & I \\
\hline 215.1281 & C11H19O4 & -0.76 & {$[\mathrm{M}-\mathrm{H}]^{-}$} & 1 \\
\hline 218.1027 & C9H16O5N & -0.69 & {$[\mathrm{M}-\mathrm{H}]^{-}$} & DL-pantothenic acid \\
\hline 218.1151 & C8H16O4N3 & 0.54 & {$[\mathrm{M}-\mathrm{H}]^{-}$} & I \\
\hline 219.0867 & C6H7N10 & 0.62 & {$[\mathrm{M}-\mathrm{H}]^{-}$} & / \\
\hline 219.1060 & $\mathrm{C} 3 \mathrm{H} 11 \mathrm{O} 2 \mathrm{~N} 10$ & -1.17 & {$[\mathrm{M}-\mathrm{H}]^{-}$} & / \\
\hline 220.0819 & C8H14O6N & -0.72 & {$[\mathrm{M}-\mathrm{H}]^{-}$} & / \\
\hline 221.1023 & C6H9N10 & 0.62 & {$[\mathrm{M}-\mathrm{H}]^{-}$} & / \\
\hline 222.0977 & $\mathrm{C} 8 \mathrm{H} 16 \mathrm{O} 6 \mathrm{~N}$ & -0.71 & {$[\mathrm{M}-\mathrm{H}]^{-}$} & / \\
\hline 225.1126 & $\mathrm{C} 12 \mathrm{H} 17 \mathrm{O} 4$ & -0.69 & {$[\mathrm{M}-\mathrm{H}]^{-}$} & / \\
\hline 227.1282 & $\mathrm{C} 12 \mathrm{H} 19 \mathrm{O} 4$ & -0.68 & {$[\mathrm{M}-\mathrm{H}]^{-}$} & / \\
\hline 227.2010 & $\mathrm{C} 14 \mathrm{H} 27 \mathrm{O} 2$ & -0.69 & {$[\mathrm{M}-\mathrm{H}]^{-}$} & Myristic acid \\
\hline 229.1075 & $\mathrm{C} 11 \mathrm{H} 17 \mathrm{O} 5$ & -0.66 & {$[\mathrm{M}-\mathrm{H}]^{-}$} & I \\
\hline 231.1232 & C11H19O5 & -0.64 & {$[\mathrm{M}-\mathrm{H}]^{-}$} & / \\
\hline 234.0977 & C9H16O6N & -0.62 & {$[\mathrm{M}-\mathrm{H}]^{-}$} & / \\
\hline 235.1011 & C3H11O3N10 & -1.03 & {$[\mathrm{M}-\mathrm{H}]^{-}$} & / \\
\hline 243.1234 & C12H19O5 & -0.52 & {$[\mathrm{M}-\mathrm{H}]^{-}$} & / \\
\hline 244.1185 & $\mathrm{C} 11 \mathrm{H} 18 \mathrm{O} 5 \mathrm{~N}$ & -0.52 & {$[\mathrm{M}-\mathrm{H}]^{-}$} & / \\
\hline 247.1181 & C11H19O6 & -0.5 & {$[\mathrm{M}-\mathrm{H}]^{-}$} & / \\
\hline 253.2168 & $\mathrm{C} 16 \mathrm{H} 29 \mathrm{O} 2$ & -0.5 & {$[\mathrm{M}-\mathrm{H}]^{-}$} & Palmitoleicacid \\
\hline 278.2247 & $\mathrm{C} 16 \mathrm{H} 28 \mathrm{ON} 3$ & 0.93 & {$[\mathrm{M}-\mathrm{H}]^{-}$} & 1 \\
\hline 280.2405 & C16H30ON3 & 1 & {$[\mathrm{M}-\mathrm{H}]^{-}$} & I \\
\hline 281.2483 & C18H33O2 & -0.33 & {$[\mathrm{M}-\mathrm{H}]^{-}$} & Oleic acid \\
\hline 282.2516 & I & I & I & / \\
\hline 285.0950 & C9H13O5N6 & -0.16 & {$[\mathrm{M}-\mathrm{H}]^{-}$} & / \\
\hline 295.2274 & $\mathrm{C} 18 \mathrm{H} 31 \mathrm{O} 3$ & -0.45 & {$[\mathrm{M}-\mathrm{H}]^{-}$} & / \\
\hline 302.2333 & $\mathrm{C} 16 \mathrm{H} 32 \mathrm{O} 4 \mathrm{~N}$ & -0.4 & {$[\mathrm{M}-\mathrm{H}]^{-}$} & / \\
\hline 303.2365 & $\mathrm{C} 10 \mathrm{H} 27 \mathrm{ON} 10$ & -0.88 & {$[\mathrm{M}-\mathrm{H}]^{-}$} & / \\
\hline 305.2330 & C16H33O5 & -0.43 & {$[\mathrm{M}-\mathrm{H}]^{-}$} & / \\
\hline 310.2146 & $\mathrm{C} 16 \mathrm{H} 28 \mathrm{O} 3 \mathrm{~N} 3$ & 0.99 & {$[\mathrm{M}-\mathrm{H}]^{-}$} & I \\
\hline 312.2303 & C16H30O3N3 & 0.96 & {$[\mathrm{M}-\mathrm{H}]^{-}$} & / \\
\hline 313.2380 & C18H33O4 & -0.37 & {$[\mathrm{M}-\mathrm{H}]^{-}$} & / \\
\hline 314.2459 & $\mathrm{C} 16 \mathrm{H} 32 \mathrm{O} 3 \mathrm{~N} 3$ & 0.95 & {$[\mathrm{M}-\mathrm{H}]^{-}$} & / \\
\hline 318.2280 & $\mathrm{C} 16 \mathrm{H} 32 \mathrm{O} 5 \mathrm{~N}$ & -0.53 & {$[\mathrm{M}-\mathrm{H}]^{-}$} & / \\
\hline 329.2329 & C18H33O5 & -0.44 & {$[\mathrm{M}-\mathrm{H}]^{-}$} & / \\
\hline 330.2647 & $\mathrm{C} 18 \mathrm{H} 36 \mathrm{O} 4 \mathrm{~N}$ & -0.45 & {$[\mathrm{M}-\mathrm{H}]^{-}$} & I \\
\hline
\end{tabular}




\begin{tabular}{lccll}
\hline 331.2484 & C18H35O5 & -0.39 & {$[\mathrm{M}-\mathrm{H}]^{-}$} & $/$ \\
333.2153 & C15H25ON8 & -0.42 & {$[\mathrm{M}-\mathrm{H}]^{-}$} & $/$ \\
343.2121 & C18H31O6 & -0.58 & {$[\mathrm{M}-\mathrm{H}]^{-}$} & $/$ \\
344.2153 & C11H30O7N5 & 0.27 & {$[\mathrm{M}-\mathrm{H}]^{-}$} & $/$ \\
427.3421 & C25H47O5 & -0.83 & {$[\mathrm{M}-\mathrm{H}]^{-}$} & $/$ \\
428.3454 & C19H42O2N9 & -1.3 & {$[\mathrm{M}-\mathrm{H}]^{-}$} & $/$ \\
453.3578 & $\mathrm{C} 27 \mathrm{H} 49 \mathrm{O} 5$ & -0.71 & {$[\mathrm{M}-\mathrm{H}]^{-}$} & $/$ \\
\hline
\end{tabular}

Table S6. Detailed information of ions classified in Group 5 in Figure 1.4-12

\begin{tabular}{|c|c|c|c|c|}
\hline$m / z$ & Composition & $\Delta \mathbf{m m u}$ & Ionmode & Compounds \\
\hline 116.0707 & $\mathrm{C} 5 \mathrm{H} 10 \mathrm{O} 2 \mathrm{~N}$ & 0.12 & {$[\mathrm{M}+\mathrm{H}]^{+}$} & 1 \\
\hline 157.0857 & $\mathrm{C} 8 \mathrm{H} 13 \mathrm{O} 3$ & -0.21 & {$[\mathrm{M}+\mathrm{H}]^{+}$} & l \\
\hline 162.1122 & $\mathrm{C} 7 \mathrm{H} 16 \mathrm{O} 3 \mathrm{~N}$ & -0.28 & {$[\mathrm{M}+\mathrm{H}]^{+}$} & l \\
\hline 187.0962 & $\mathrm{C} 9 \mathrm{H} 15 \mathrm{O} 4$ & -0.35 & {$[\mathrm{M}+\mathrm{H}]^{+}$} & l \\
\hline 190.1071 & $\mathrm{C} 8 \mathrm{H} 16 \mathrm{O} 4 \mathrm{~N}$ & -0.35 & {$[\mathrm{M}+\mathrm{H}]^{+}$} & l \\
\hline 190.1434 & $\mathrm{C} 9 \mathrm{H} 20 \mathrm{O} 3 \mathrm{~N}$ & -0.39 & {$[\mathrm{M}+\mathrm{H}]^{+}$} & l \\
\hline 191.1409 & C8H19O3N2 & 1.47 & {$[\mathrm{M}+\mathrm{H}]^{+}$} & l \\
\hline 191.1467 & C3H15N10 & -0.86 & {$[\mathrm{M}+\mathrm{H}]^{+}$} & l \\
\hline 192.1475 & I & I & I & I \\
\hline 192.1500 & $\mathrm{C} 11 \mathrm{H} 18 \mathrm{~N} 3$ & 0.55 & {$[\mathrm{M}+\mathrm{H}]^{+}$} & l \\
\hline 194.1384 & $\mathrm{C} 8 \mathrm{H} 20 \mathrm{O} 4 \mathrm{~N}$ & -0.35 & {$[\mathrm{M}+\mathrm{H}]^{+}$} & l \\
\hline 199.1325 & C11H19O3 & -0.4 & {$[\mathrm{M}+\mathrm{H}]^{+}$} & I \\
\hline 204.1227 & C9H18O4N & -0.36 & {$[\mathrm{M}+\mathrm{H}]^{+}$} & l \\
\hline 204.1590 & $\mathrm{C} 10 \mathrm{H} 22 \mathrm{O} 3 \mathrm{~N}$ & -0.38 & {$[\mathrm{M}+\mathrm{H}]^{+}$} & l \\
\hline 218.1382 & $\mathrm{C} 10 \mathrm{H} 20 \mathrm{O} 4 \mathrm{~N}$ & -0.47 & {$[\mathrm{M}+\mathrm{H}]^{+}$} & l \\
\hline 220.1539 & $\mathrm{C} 10 \mathrm{H} 22 \mathrm{O} 4 \mathrm{~N}$ & -0.45 & {$[\mathrm{M}+\mathrm{H}]^{+}$} & l \\
\hline 232.1538 & $\mathrm{C} 11 \mathrm{H} 22 \mathrm{O} 4 \mathrm{~N}$ & -0.5 & {$[\mathrm{M}+\mathrm{H}]^{+}$} & l \\
\hline 234.1694 & $\mathrm{C} 11 \mathrm{H} 24 \mathrm{O} 4 \mathrm{~N}$ & -0.55 & {$[\mathrm{M}+\mathrm{H}]^{+}$} & l \\
\hline 246.1694 & $\mathrm{C} 12 \mathrm{H} 24 \mathrm{O} 4 \mathrm{~N}$ & -0.53 & {$[\mathrm{M}+\mathrm{H}]^{+}$} & l \\
\hline 247.1728 & C6H19ON10 & -1.04 & {$[\mathrm{M}+\mathrm{H}]^{+}$} & l \\
\hline 274.2005 & $\mathrm{C} 14 \mathrm{H} 28 \mathrm{O} 4 \mathrm{~N}$ & -0.79 & {$[\mathrm{M}+\mathrm{H}]^{+}$} & l \\
\hline 123.0802 & C8H11O & -1.3 & {$[\mathrm{M}-\mathrm{H}]^{-}$} & I \\
\hline 141.0909 & $\mathrm{C} 8 \mathrm{H} 13 \mathrm{O} 2$ & -1.18 & {$[\mathrm{M}-\mathrm{H}]^{-}$} & Octenoic acid \\
\hline 143.1066 & $\mathrm{C} 8 \mathrm{H} 15 \mathrm{O} 2$ & -1.17 & {$[\mathrm{M}-\mathrm{H}]^{-}$} & Octanoic acid \\
\hline 157.0859 & C8H13O3 & -1.1 & {$[\mathrm{M}-\mathrm{H}]^{-}$} & I \\
\hline 157.1223 & $\mathrm{C} 9 \mathrm{H} 17 \mathrm{O} 2$ & -1.11 & {$[\mathrm{M}-\mathrm{H}]^{-}$} & Nonanoic acid \\
\hline 158.0892 & C2H8N9 & -1.59 & {$[\mathrm{M}-\mathrm{H}]^{-}$} & I \\
\hline 158.1256 & I & I & I & l \\
\hline 159.1016 & C8H15O3 & -1.1 & {$[\mathrm{M}-\mathrm{H}]^{-}$} & l \\
\hline 160.1049 & I & I & I & l \\
\hline 167.0701 & $\mathrm{C} 9 \mathrm{H} 11 \mathrm{O} 3$ & -1.1 & {$[\mathrm{M}-\mathrm{H}]^{-}$} & l \\
\hline
\end{tabular}




\begin{tabular}{|c|c|c|c|c|}
\hline 170.8702 & / & I & / & I \\
\hline 171.0654 & C8H11O4 & -0.89 & {$[\mathrm{M}-\mathrm{H}]^{-}$} & / \\
\hline 171.1017 & $\mathrm{C} 9 \mathrm{H} 15 \mathrm{O} 3$ & -0.98 & {$[\mathrm{M}-\mathrm{H}]^{-}$} & 9-oxononanoic acid \\
\hline 171.1379 & $\mathrm{C} 10 \mathrm{H} 19 \mathrm{O} 2$ & -1.15 & {$[\mathrm{M}-\mathrm{H}]^{-}$} & I \\
\hline 171.3326 & I & I & I & / \\
\hline 172.1050 & C3H10N9 & -1.43 & {$[\mathrm{M}-\mathrm{H}]^{-}$} & / \\
\hline 173.0809 & C8H13O4 & -1.02 & {$[\mathrm{M}-\mathrm{H}]^{-}$} & / \\
\hline 173.1059 & C6H13O2N4 & 1.53 & {$[\mathrm{M}-\mathrm{H}]^{-}$} & / \\
\hline 173.1084 & C11H13N2 & -0.07 & {$[\mathrm{M}-\mathrm{H}]^{-}$} & / \\
\hline 173.1173 & C9H17O3 & -1.05 & {$[\mathrm{M}-\mathrm{H}]^{-}$} & / \\
\hline 174.0887 & C6H12O3N3 & 0.31 & {$[\mathrm{M}-\mathrm{H}]^{-}$} & / \\
\hline 175.0966 & C8H15O4 & -1.03 & {$[\mathrm{M}-\mathrm{H}]^{-}$} & / \\
\hline 185.0810 & C9H13O4 & -0.91 & {$[\mathrm{M}-\mathrm{H}]^{-}$} & I \\
\hline 187.0967 & C9H15O4 & -0.9 & {$[\mathrm{M}-\mathrm{H}]^{-}$} & Azelaic acid \\
\hline 188.1000 & C3H10ON9 & -1.38 & {$[\mathrm{M}-\mathrm{H}]^{-}$} & I \\
\hline 188.1044 & $\mathrm{C} 7 \mathrm{H} 14 \mathrm{O} 3 \mathrm{~N} 3$ & 0.37 & {$[\mathrm{M}-\mathrm{H}]^{-}$} & / \\
\hline 189.0759 & C8H13O5 & -0.93 & {$[\mathrm{M}-\mathrm{H}]^{-}$} & / \\
\hline 189.1123 & C9H17O4 & -0.96 & {$[\mathrm{M}-\mathrm{H}]^{-}$} & / \\
\hline 190.0837 & C6H12O4N3 & 0.4 & {$[\mathrm{M}-\mathrm{H}]^{-}$} & / \\
\hline 190.1202 & C7H16O3N3 & 0.4 & {$[\mathrm{M}-\mathrm{H}]^{-}$} & / \\
\hline 191.1079 & $\mathrm{C} 12 \mathrm{H} 15 \mathrm{O} 2$ & 0.25 & {$[\mathrm{M}-\mathrm{H}]^{-}$} & / \\
\hline 197.1175 & $\mathrm{C} 11 \mathrm{H} 17 \mathrm{O} 3$ & -0.87 & {$[\mathrm{M}-\mathrm{H}]^{-}$} & / \\
\hline 198.1208 & C4H16O4N5 & 0.04 & {$[\mathrm{M}-\mathrm{H}]^{-}$} & / \\
\hline 199.1331 & C11H19O3 & -0.87 & {$[\mathrm{M}-\mathrm{H}]^{-}$} & / \\
\hline 199.1694 & $\mathrm{C} 12 \mathrm{H} 23 \mathrm{O} 2$ & -0.84 & {$[\mathrm{M}-\mathrm{H}]^{-}$} & / \\
\hline 201.0759 & C9H13O5 & -0.86 & {$[\mathrm{M}-\mathrm{H}]^{-}$} & / \\
\hline 202.0838 & C7H12O4N3 & 0.5 & {$[\mathrm{M}-\mathrm{H}]^{-}$} & / \\
\hline 203.0917 & C9H15O5 & -0.81 & {$[\mathrm{M}-\mathrm{H}]^{-}$} & / \\
\hline 204.0995 & C7H14O4N3 & 0.53 & {$[\mathrm{M}-\mathrm{H}]^{-}$} & / \\
\hline 205.1029 & C16H13 & 0.61 & {$[\mathrm{M}-\mathrm{H}]^{-}$} & / \\
\hline 217.0710 & C6H5N10 & 0.63 & {$[\mathrm{M}-\mathrm{H}]^{-}$} & / \\
\hline 234.0738 & C8H8O2N7 & -0.63 & {$[\mathrm{M}-\mathrm{H}]^{-}$} & / \\
\hline 241.2167 & $\mathrm{C} 15 \mathrm{H} 29 \mathrm{O} 2$ & -0.57 & {$[\mathrm{M}-\mathrm{H}]^{-}$} & / \\
\hline 250.0927 & $\mathrm{C} 9 \mathrm{H} 16 \mathrm{O} 7 \mathrm{~N}$ & -0.52 & {$[\mathrm{M}-\mathrm{H}]^{-}$} & I \\
\hline 255.2326 & $\mathrm{C} 16 \mathrm{H} 31 \mathrm{O} 2$ & -0.38 & {$[\mathrm{M}-\mathrm{H}]^{-}$} & Palmitic acid \\
\hline 256.2359 & I & I & / & / \\
\hline 257.2394 & I & I & / & / \\
\hline 265.0799 & C8H9O3N8 & -0.33 & {$[\mathrm{M}-\mathrm{H}]^{-}$} & / \\
\hline 269.2119 & $\mathrm{C} 16 \mathrm{H} 29 \mathrm{O} 3$ & -0.37 & {$[\mathrm{M}-\mathrm{H}]^{-}$} & / \\
\hline 271.2275 & $\mathrm{C} 16 \mathrm{H} 31 \mathrm{O} 3$ & -0.34 & {$[\mathrm{M}-\mathrm{H}]^{-}$} & / \\
\hline 275.2387 & $\mathrm{C} 19 \mathrm{H} 31 \mathrm{O}$ & 0.7 & {$[\mathrm{M}-\mathrm{H}]^{-}$} & / \\
\hline 283.2639 & $\mathrm{C} 18 \mathrm{H} 35 \mathrm{O} 2$ & -0.41 & {$[\mathrm{M}-\mathrm{H}]^{-}$} & Stearic acid \\
\hline 284.2673 & I & I & I & / \\
\hline 285.2069 & $\mathrm{C} 16 \mathrm{H} 29 \mathrm{O} 4$ & -0.39 & {$[\mathrm{M}-\mathrm{H}]^{-}$} & I \\
\hline
\end{tabular}




\begin{tabular}{lcccc}
\hline 286.2146 & $\mathrm{C} 14 \mathrm{H} 28 \mathrm{O} 3 \mathrm{~N} 3$ & 1.01 & {$[\mathrm{M}-\mathrm{H}]^{-}$} & $/$ \\
287.2179 & $/$ & $/$ & $/$ & $/$ \\
287.2225 & $\mathrm{C} 16 \mathrm{H} 31 \mathrm{O} 4$ & -0.28 & {$[\mathrm{M}-\mathrm{H}]^{-}$} & $/$ \\
288.2303 & $\mathrm{C} 14 \mathrm{H} 30 \mathrm{O} 3 \mathrm{~N} 3$ & 0.99 & {$[\mathrm{M}-\mathrm{H}]^{-}$} & $/$ \\
289.2337 & $/$ & $/$ & $/$ & $/$ \\
297.2430 & $\mathrm{C} 18 \mathrm{H} 33 \mathrm{O} 3$ & -0.49 & {$[\mathrm{M}-\mathrm{H}]^{-}$} & $/$ \\
349.2100 & $\mathrm{C} 15 \mathrm{H} 25 \mathrm{O} 2 \mathrm{~N} 8$ & -0.53 & {$[\mathrm{M}-\mathrm{H}]^{-}$} & $/$ \\
\hline
\end{tabular}

81

Table S7. Detailed information of ions classified in Group 6 in Figure 1.4-12

\begin{tabular}{|c|c|c|c|c|}
\hline$m / z$ & Composition & $\Delta \mathbf{m m u}$ & Ionmode & Compounds \\
\hline 60.0816 & $\mathrm{C} 3 \mathrm{H} 10 \mathrm{~N}$ & 0.79 & {$[\mathrm{M}+\mathrm{H}]^{+}$} & Trimethylamine \\
\hline 74.0970 & $\mathrm{C} 4 \mathrm{H} 12 \mathrm{~N}$ & 0.59 & {$[\mathrm{M}+\mathrm{H}]^{+}$} & I \\
\hline 75.0574 & I & I & I & I \\
\hline 79.0218 & I & I & I & I \\
\hline 91.0544 & $\mathrm{C} 7 \mathrm{H} 7$ & 0.17 & {$[\mathrm{M}+\mathrm{H}]^{+}$} & I \\
\hline 92.0841 & / & / & I & / \\
\hline 92.0904 & I & I & I & / \\
\hline 108.0810 & C7H10N & 0.23 & {$[\mathrm{M}+\mathrm{H}]^{+}$} & I \\
\hline 119.1180 & C5H15ON2 & 0.09 & {$[\mathrm{M}+\mathrm{H}]^{+}$} & / \\
\hline 128.1433 & $\mathrm{C} 8 \mathrm{H} 18 \mathrm{~N}$ & -0.03 & {$[\mathrm{M}+\mathrm{H}]^{+}$} & / \\
\hline 129.1467 & I & I & I & / \\
\hline 133.0759 & C8H9N2 & -0.1 & {$[\mathrm{M}+\mathrm{H}]^{+}$} & / \\
\hline 133.1335 & C6H17ON2 & -0.1 & {$[\mathrm{M}+\mathrm{H}]^{+}$} & / \\
\hline 134.0963 & $\mathrm{C} 9 \mathrm{H} 12 \mathrm{~N}$ & -0.08 & {$[\mathrm{M}+\mathrm{H}]^{+}$} & / \\
\hline 135.1127 & $\mathrm{C} 5 \mathrm{H} 15 \mathrm{O} 2 \mathrm{~N} 2$ & -0.15 & {$[\mathrm{M}+\mathrm{H}]^{+}$} & / \\
\hline 137.1153 & C2H13ON6 & 0.75 & {$[\mathrm{M}+\mathrm{H}]^{+}$} & / \\
\hline 138.1123 & I & I & I & / \\
\hline 152.1279 & I & I & I & / \\
\hline 166.1435 & / & / & / & I \\
\hline 167.1469 & / & / & I & I \\
\hline 176.1278 & $\mathrm{C} 8 \mathrm{H} 18 \mathrm{O} 3 \mathrm{~N}$ & -0.32 & {$[\mathrm{M}+\mathrm{H}]^{+}$} & I \\
\hline 177.1311 & $\mathrm{C} 2 \mathrm{H} 13 \mathrm{~N} 10$ & -0.81 & {$[\mathrm{M}+\mathrm{H}]^{+}$} & / \\
\hline 178.1070 & $\mathrm{C} 7 \mathrm{H} 16 \mathrm{O} 4 \mathrm{~N}$ & -0.34 & {$[\mathrm{M}+\mathrm{H}]^{+}$} & / \\
\hline 180.1591 & 1 & I & I & / \\
\hline 181.1624 & $\mathrm{C} 2 \mathrm{H} 17 \mathrm{~N} 10$ & -0.81 & {$[\mathrm{M}+\mathrm{H}]^{+}$} & / \\
\hline 189.1118 & С9H17O4 & -0.37 & {$[\mathrm{M}+\mathrm{H}]^{+}$} & / \\
\hline 192.1226 & $\mathrm{C} 8 \mathrm{H} 18 \mathrm{O} 4 \mathrm{~N}$ & -0.44 & {$[\mathrm{M}+\mathrm{H}]^{+}$} & / \\
\hline 201.1957 & $\mathrm{C} 11 \mathrm{H} 25 \mathrm{ON} 2$ & -0.45 & {$[\mathrm{M}+\mathrm{H}]^{+}$} & / \\
\hline 206.1383 & C9H20O4N & -0.41 & {$[\mathrm{M}+\mathrm{H}]^{+}$} & / \\
\hline 208.1539 & $\mathrm{C} 9 \mathrm{H} 22 \mathrm{O} 4 \mathrm{~N}$ & -0.44 & {$[\mathrm{M}+\mathrm{H}]^{+}$} & / \\
\hline 209.1644 & $\mathrm{C} 12 \mathrm{H} 21 \mathrm{ON} 2$ & -0.44 & {$[\mathrm{M}+\mathrm{H}]^{+}$} & I \\
\hline
\end{tabular}




\begin{tabular}{|c|c|c|c|c|}
\hline 220.1175 & $\mathrm{C} 9 \mathrm{H} 18 \mathrm{O} 5 \mathrm{~N}$ & -0.5 & {$[\mathrm{M}+\mathrm{H}]^{+}$} & I \\
\hline 222.1331 & $\mathrm{C} 9 \mathrm{H} 20 \mathrm{O} 5 \mathrm{~N}$ & -0.49 & {$[\mathrm{M}+\mathrm{H}]^{+}$} & / \\
\hline 224.1851 & I & / & I & / \\
\hline 234.1332 & $\mathrm{C} 10 \mathrm{H} 20 \mathrm{O} 5 \mathrm{~N}$ & -0.53 & {$[\mathrm{M}+\mathrm{H}]^{+}$} & I \\
\hline 234.2060 & $\mathrm{C} 12 \mathrm{H} 28 \mathrm{O} 3 \mathrm{~N}$ & -0.5 & {$[\mathrm{M}+\mathrm{H}]^{+}$} & / \\
\hline 248.1486 & $\mathrm{C} 11 \mathrm{H} 22 \mathrm{O} 5 \mathrm{~N}$ & -0.6 & {$[\mathrm{M}+\mathrm{H}]^{+}$} & / \\
\hline 262.1642 & $\mathrm{C} 12 \mathrm{H} 24 \mathrm{O} 5 \mathrm{~N}$ & -0.65 & {$[\mathrm{M}+\mathrm{H}]^{+}$} & I \\
\hline 263.1958 & $\mathrm{C} 12 \mathrm{H} 27 \mathrm{O} 4 \mathrm{~N} 2$ & -0.62 & {$[\mathrm{M}+\mathrm{H}]^{+}$} & / \\
\hline 288.2162 & C15H30O4N & -0.78 & {$[\mathrm{M}+\mathrm{H}]^{+}$} & / \\
\hline 288.2527 & $\mathrm{C} 16 \mathrm{H} 34 \mathrm{O} 3 \mathrm{~N}$ & -0.79 & {$[\mathrm{M}+\mathrm{H}]^{+}$} & I \\
\hline 304.2474 & $\mathrm{C} 16 \mathrm{H} 34 \mathrm{O} 4 \mathrm{~N}$ & -0.81 & {$[\mathrm{M}+\mathrm{H}]^{+}$} & / \\
\hline 306.2268 & $\mathrm{C} 15 \mathrm{H} 32 \mathrm{O} 5 \mathrm{~N}$ & -0.78 & {$[\mathrm{M}+\mathrm{H}]^{+}$} & I \\
\hline 316.2840 & C18H38O3N & -0.75 & {$[\mathrm{M}+\mathrm{H}]^{+}$} & / \\
\hline 391.2833 & C24H39O4 & -0.92 & {$[\mathrm{M}+\mathrm{H}]^{+}$} & / \\
\hline 462.1457 & $\mathrm{C} 15 \mathrm{H} 28 \mathrm{O} 15 \mathrm{~N}$ & 0.31 & {$[\mathrm{M}+\mathrm{H}]^{+}$} & / \\
\hline 59.0125 & $\mathrm{C} 2 \mathrm{H} 3 \mathrm{O} 2$ & -1.33 & {$[\mathrm{M}-\mathrm{H}]^{-}$} & I \\
\hline 60.9875 & & & {$[\mathrm{M}-\mathrm{H}]^{-}$} & I \\
\hline 62.9960 & & & {$[\mathrm{M}-\mathrm{H}]^{-}$} & / \\
\hline 73.0281 & $\mathrm{C} 3 \mathrm{H} 5 \mathrm{O} 2$ & -1.39 & {$[\mathrm{M}-\mathrm{H}]^{-}$} & / \\
\hline 75.9788 & & & {$[\mathrm{M}-\mathrm{H}]^{-}$} & / \\
\hline 77.9900 & & & {$[\mathrm{M}-\mathrm{H}]^{-}$} & / \\
\hline 80.9980 & $\mathrm{C} 4 \mathrm{HO} 2$ & -0.26 & {$[\mathrm{M}-\mathrm{H}]^{-}$} & I \\
\hline 87.0074 & $\mathrm{C} 3 \mathrm{H} 3 \mathrm{O} 3$ & -1.4 & {$[\mathrm{M}-\mathrm{H}]^{-}$} & I \\
\hline 87.0437 & I & I & I & I \\
\hline 88.0107 & I & I & I & I \\
\hline 89.0230 & $\mathrm{C} 3 \mathrm{H} 5 \mathrm{O} 3$ & -1.4 & {$[\mathrm{M}-\mathrm{H}]^{-}$} & I \\
\hline 89.9945 & I & I & I & I \\
\hline 90.0264 & 1 & / & I & / \\
\hline 91.0207 & $\mathrm{C} 6 \mathrm{H} 3 \mathrm{O}$ & 1.74 & {$[\mathrm{M}-\mathrm{H}]^{-}$} & I \\
\hline 92.0101 & I & I & I & I \\
\hline 93.9894 & I & / & l & I \\
\hline 122.0207 & $\mathrm{CH} 4 \mathrm{O} 4 \mathrm{~N} 3$ & 0.04 & {$[\mathrm{M}-\mathrm{H}]^{-}$} & / \\
\hline 122.9796 & & & {$[\mathrm{M}-\mathrm{H}]^{-}$} & I \\
\hline 126.9871 & СH3O7 & -1.29 & {$[\mathrm{M}-\mathrm{H}]^{-}$} & I \\
\hline 152.0189 & $\mathrm{C} 3 \mathrm{H} 6 \mathrm{O} 6 \mathrm{~N}$ & -1.15 & {$[\mathrm{M}-\mathrm{H}]^{-}$} & / \\
\hline 165.0397 & C2HN10 & 0.62 & {$[\mathrm{M}-\mathrm{H}]^{-}$} & / \\
\hline 165.9847 & I & I & I & / \\
\hline 173.9771 & $\mathrm{C} 3 \mathrm{O} 6 \mathrm{~N} 3$ & -2.12 & {$[\mathrm{M}-\mathrm{H}]^{-}$} & / \\
\hline 177.0911 & $\mathrm{C} 11 \mathrm{H} 13 \mathrm{O} 2$ & -1.07 & {$[\mathrm{M}-\mathrm{H}]^{-}$} & I \\
\hline 182.9876 & $\mathrm{C} 2 \mathrm{H} 3 \mathrm{O} 8 \mathrm{~N} 2$ & -1.85 & {$[\mathrm{M}-\mathrm{H}]^{-}$} & / \\
\hline 197.9748 & I & I & I & / \\
\hline 232.9847 & C9HO6N2 & 0.75 & {$[\mathrm{M}-\mathrm{H}]^{-}$} & / \\
\hline 239.0590 & I & I & I & I \\
\hline 261.1340 & $\mathrm{C} 12 \mathrm{H} 21 \mathrm{O} 6$ & -0.41 & {$[\mathrm{M}-\mathrm{H}]^{-}$} & I \\
\hline
\end{tabular}




\begin{tabular}{lllll}
\hline 265.9827 & $\mathrm{C} 12 \mathrm{O} 5 \mathrm{~N} 3$ & -1.62 & {$[\mathrm{M}-\mathrm{H}]^{-}$} & $/$ \\
\hline
\end{tabular}

Table S8. Detailed information of enhancement ratios (ER) of identified compounds.

\begin{tabular}{|c|c|c|c|c|}
\hline$m / z$ & Compounds & $\begin{array}{c}\text { Intensity(t0) } \\
\text { (a.u.) }\end{array}$ & $\begin{array}{c}\text { Intensity(max) } \\
\text { (a.u.) }\end{array}$ & $\begin{array}{c}\text { Enhancement } \\
\text { ratios } \\
\end{array}$ \\
\hline \multicolumn{5}{|c|}{ Unsaturated aldehydes } \\
\hline 85.0653 & Pentenal & $1.20 \mathrm{E} 5$ & 1.98E6 & 16.5 \\
\hline 111.0806 & Heptadienal & 3.89E4 & 3.83E5 & 9.8 \\
\hline 127.1118 & Octenal & $2.55 \mathrm{E} 5$ & 2.18E6 & 8.5 \\
\hline 139.1116 & Nonadienal & $3.84 \mathrm{E} 5$ & 2.79E6 & 7.3 \\
\hline 141.1272 & Nonenal & $2.09 \mathrm{E} 5$ & 9.87E5 & 4.7 \\
\hline 153.1271 & Decadienal & 2.74E5 & 4.25E7 & 155.1 \\
\hline 155.1427 & Decenal & $1.03 \mathrm{E} 5$ & $3.60 \mathrm{E} 5$ & 3.5 \\
\hline \multicolumn{5}{|c|}{ Unsaturated fatty acids } \\
\hline 71.0125 & Acrylic acid & 8.45E5 & 9.83E6 & 11.6 \\
\hline 113.0595 & Hexenoic acid & $3.28 \mathrm{E} 4$ & 3.17E5 & 9.7 \\
\hline 127.0752 & Heptenoic acid & $2.24 \mathrm{E} 4$ & $1.50 \mathrm{E} 6$ & 67.0 \\
\hline 141.0909 & Octenoic acid & $5.65 \mathrm{E} 4$ & $1.88 \mathrm{E} 6$ & 33.3 \\
\hline 155.1066 & Nonenoic acid & $3.78 \mathrm{E} 4$ & 3.07E6 & 81.2 \\
\hline 169.1224 & Decenoic Acid & $1.54 \mathrm{E} 4$ & $5.68 \mathrm{E} 6$ & 368.8 \\
\hline 183.1381 & Undecylenic acid & $1.69 \mathrm{E} 3$ & 2.99E5 & 176.9 \\
\hline 253.2168 & Palmitoleic acid & $1.13 \mathrm{E} 3$ & 2.07E5 & 183.2 \\
\hline 279.2326 & Linoleic acid & 0 & 9.02E6 & N.A. \\
\hline 281.2482 & Oleic acid & 0 & $1.52 \mathrm{E} 7$ & N.A. \\
\hline \multicolumn{5}{|c|}{ Saturated fatty acids } \\
\hline 115.0751 & Hexanoic acid & $6.52 \mathrm{E} 5$ & $6.26 \mathrm{E} 6$ & 9.6 \\
\hline 129.0908 & Heptanoic acid & $2.26 \mathrm{E} 5$ & $1.94 \mathrm{E} 6$ & 8.6 \\
\hline 143.1066 & Octanoic acid & $1.36 \mathrm{E} 6$ & 2.77E6 & 2.0 \\
\hline 157.1223 & Nonanoic Acid & $4.18 \mathrm{E} 5$ & $5.42 \mathrm{E} 6$ & 13.0 \\
\hline 227.2010 & Myristic acid & $3.75 \mathrm{E} 4$ & $3.44 \mathrm{E} 5$ & 9.2 \\
\hline 255.2326 & Palmitic acid & $2.80 \mathrm{E} 5$ & 2.99E7 & 106.8 \\
\hline 283.2638 & Stearic acid & $1.25 \mathrm{E} 4$ & $1.20 \mathrm{E} 6$ & 96.0 \\
\hline \multicolumn{5}{|c|}{ N-containing heterocyclic compounds } \\
\hline 109.0763 & Dimethylpyrazine & 1.70E6 & 3.60E6 & 2.1 \\
\hline 110.0603 & 3-methoxy-4-pyridine & 4.03E5 & $1.30 \mathrm{E} 6$ & 3.2 \\
\hline 121.0761 & 2-vinyl-6-methylpyrazine & $2.50 \mathrm{E} 5$ & 8.54E5 & 3.4 \\
\hline 123.0917 & Trimethylpyrazine & 3.34E6 & $1.00 \mathrm{E} 7$ & 3.0 \\
\hline 125.0710 & $\begin{array}{c}\text { 5-methyl-2-pyrazine } \\
\text { methanol }\end{array}$ & $5.28 \mathrm{E} 4$ & $5.13 \mathrm{E} 5$ & 9.7 \\
\hline 128.1070 & $\mathrm{~N}$-acetyl piperidine & $1.03 \mathrm{E} 5$ & $6.57 \mathrm{E} 5$ & 6.4 \\
\hline 135.0916 & 2-methyl-5- & $4.59 \mathrm{E} 5$ & 2.89E6 & 6.3 \\
\hline
\end{tabular}




\begin{tabular}{|c|c|c|c|c|}
\hline \multicolumn{5}{|c|}{ propenylpyrazine } \\
\hline 137.0708 & $\begin{array}{c}\text { 2-acetyl-3- } \\
\text { methylpyrazine }\end{array}$ & $4.68 \mathrm{E} 4$ & $3.80 \mathrm{E} 5$ & 8.1 \\
\hline 137.1072 & $\begin{array}{l}\text { 2,5-dimethyl-3- } \\
\text { ethylpyrazine }\end{array}$ & $1.84 \mathrm{E} 6$ & $8.11 \mathrm{E} 6$ & 4.4 \\
\hline 138.0548 & $\begin{array}{l}\text { Methyl pyridine-3- } \\
\text { carboxylate }\end{array}$ & $3.11 \mathrm{E} 5$ & $1.80 \mathrm{E} 6$ & 5.8 \\
\hline 151.1227 & $\begin{array}{l}\text { 2,6-diethyl-3- } \\
\text { methylpyrazine }\end{array}$ & $2.48 \mathrm{E} 5$ & $1.35 \mathrm{E} 6$ & 5.4 \\
\hline \multicolumn{5}{|c|}{ O-containing heterocyclic compounds } \\
\hline 125.0597 & 2-acetyl-5-methylfuran & $6.27 \mathrm{E} 4$ & $3.02 \mathrm{E} 5$ & 4.8 \\
\hline 129.0910 & $\begin{array}{l}\text { 5-propyldihydro-2(3H)- } \\
\text { furanone }\end{array}$ & $2.26 \mathrm{E} 5$ & $1.22 \mathrm{E} 6$ & 5.4 \\
\hline 143.1066 & $\begin{array}{l}\text { 5-butyldihydro-2(3H)- } \\
\text { furanone }\end{array}$ & $3.79 \mathrm{E} 5$ & 6.37E6 & 16.8 \\
\hline 171.1377 & $\begin{array}{l}\text { 5-pentyldihydro-2(3H)- } \\
\text { furanone }\end{array}$ & $1.76 \mathrm{E} 5$ & $1.05 \mathrm{E} 6$ & 6.0 \\
\hline 185.1533 & $\begin{array}{l}\text { 5-heptyldihydro-2(3H)- } \\
\text { furanone }\end{array}$ & $5.99 \mathrm{E} 4$ & $6.53 \mathrm{E} 5$ & 10.9 \\
\hline 227.2000 & $\begin{array}{l}\text { 5-decyldihydro-2(3H)- } \\
\text { furanone }\end{array}$ & $4.68 \mathrm{E} 3$ & $2.89 \mathrm{E} 5$ & 61.8 \\
\hline \multicolumn{5}{|c|}{ Others } \\
\hline 60.0816 & Trimethylamine & $1.79 \mathrm{E} 5$ & 9.79E6 & 54.7 \\
\hline 121.0282 & Benzoic acid & 3.34E6 & $1.96 \mathrm{E} 7$ & 5.9 \\
\hline 122.0601 & $\mathrm{C}_{7} \mathrm{H}_{7} \mathrm{ON}$ & $1.01 \mathrm{E} 6$ & $1.48 \mathrm{E} 7$ & 14.7 \\
\hline 133.1335 & $\mathrm{C}_{6} \mathrm{H}_{16} \mathrm{ON}_{2}$ & $1.90 \mathrm{E} 4$ & $1.81 \mathrm{E} 6$ & 95.3 \\
\hline 171.1017 & 9-oxononanoic acid & $5.20 \mathrm{E} 5$ & $1.08 \mathrm{E} 8$ & 207.7 \\
\hline 187.0967 & Azelaic acid & $2.46 \mathrm{E} 5$ & $1.12 \mathrm{E} 7$ & 45.5 \\
\hline 190.1434 & $\mathrm{C}_{9} \mathrm{H}_{19} \mathrm{O}_{3} \mathrm{~N}$ & 5.04E5 & $8.78 \mathrm{E} 7$ & 174.2 \\
\hline 218.1027 & DL-pantothenic acid & $3.43 \mathrm{E} 4$ & $1.14 \mathrm{E} 7$ & 332.4 \\
\hline 226.1797 & $\mathrm{C}_{13} \mathrm{H}_{23} \mathrm{O}_{2} \mathrm{~N}$ & $1.30 \mathrm{E} 5$ & 3.72E7 & 286.2 \\
\hline
\end{tabular}




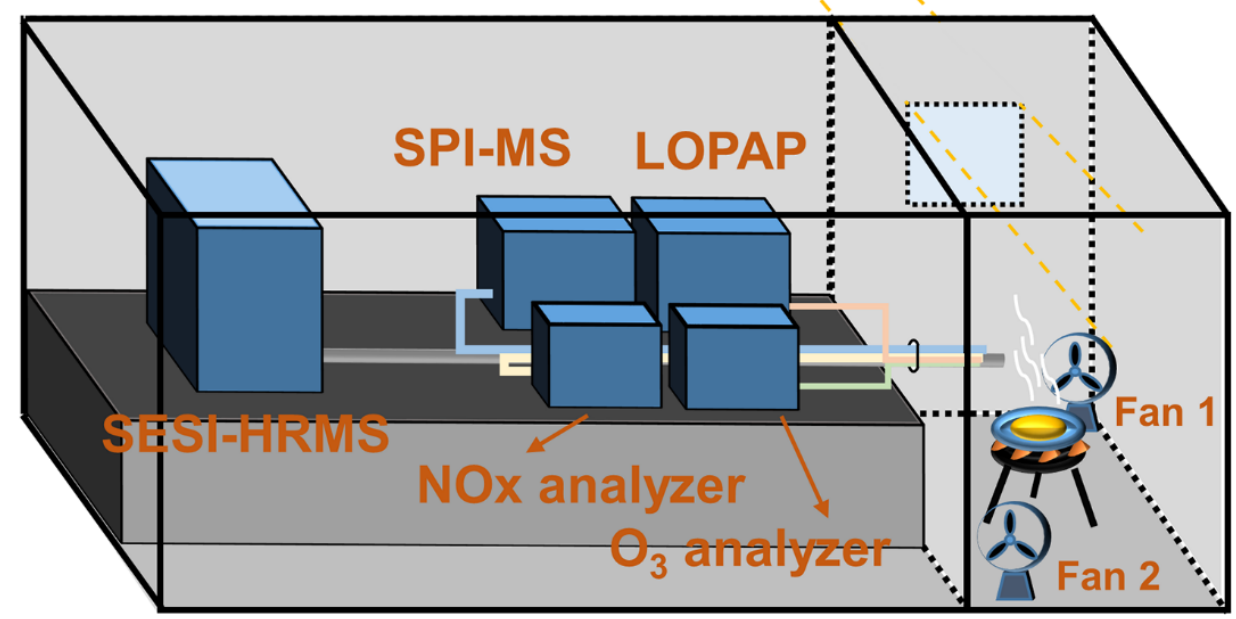

Figure S1. Schematic of the cooking experiments
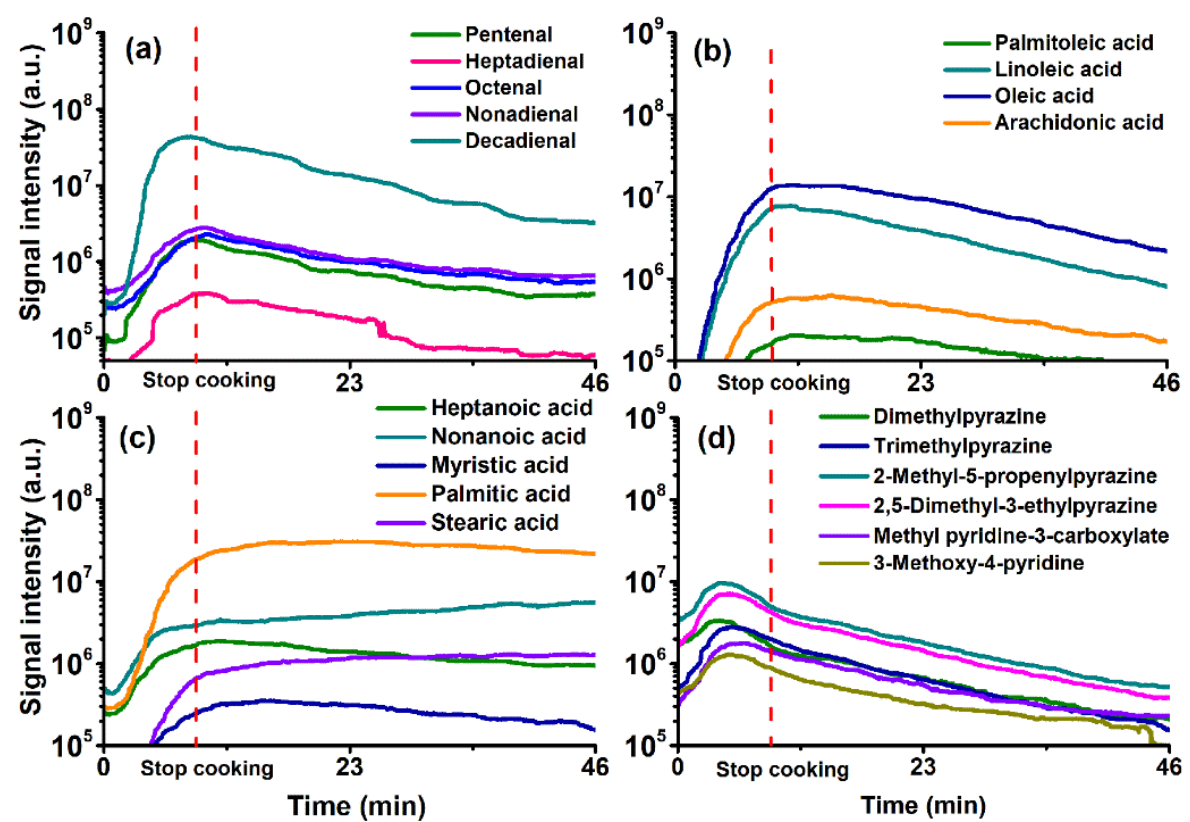

91 Figure S2. Time-signal intensity profiles of (a) unsaturated aldehydes, (b) unsaturated fatty acids, (c) saturated fatty acids and (d) N-containing heterocyclic compounds detected during peanut oil cooking, which are consistent with those reported in previous studies ${ }^{6,8,10}$. 
(a)

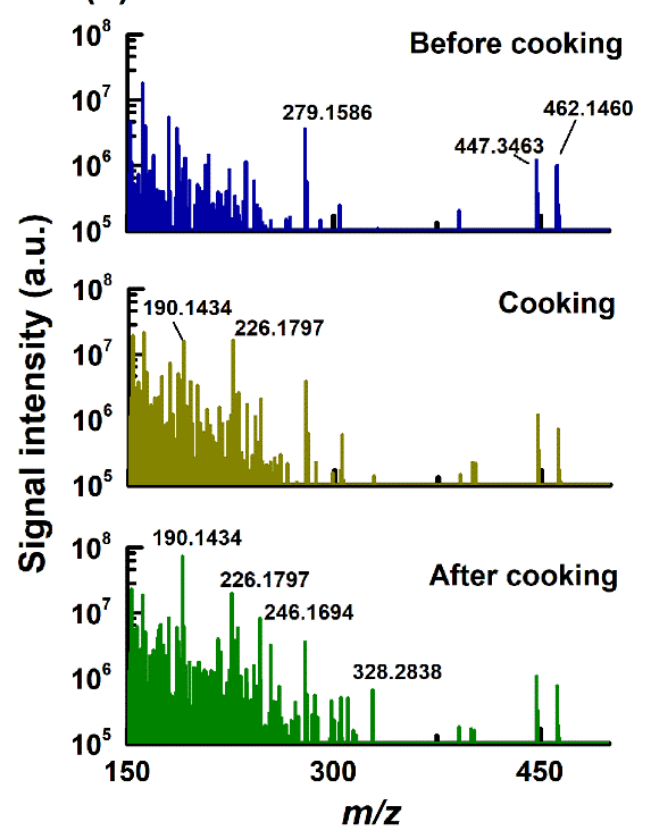

(b)

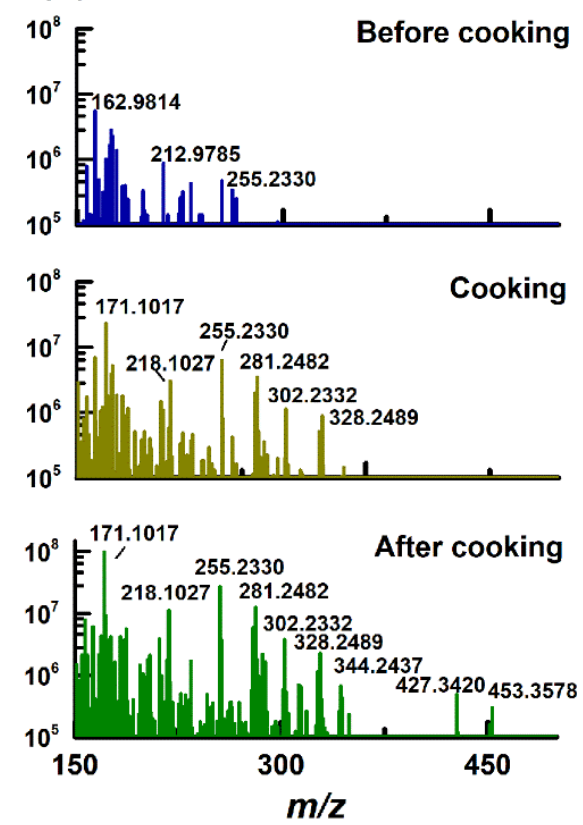

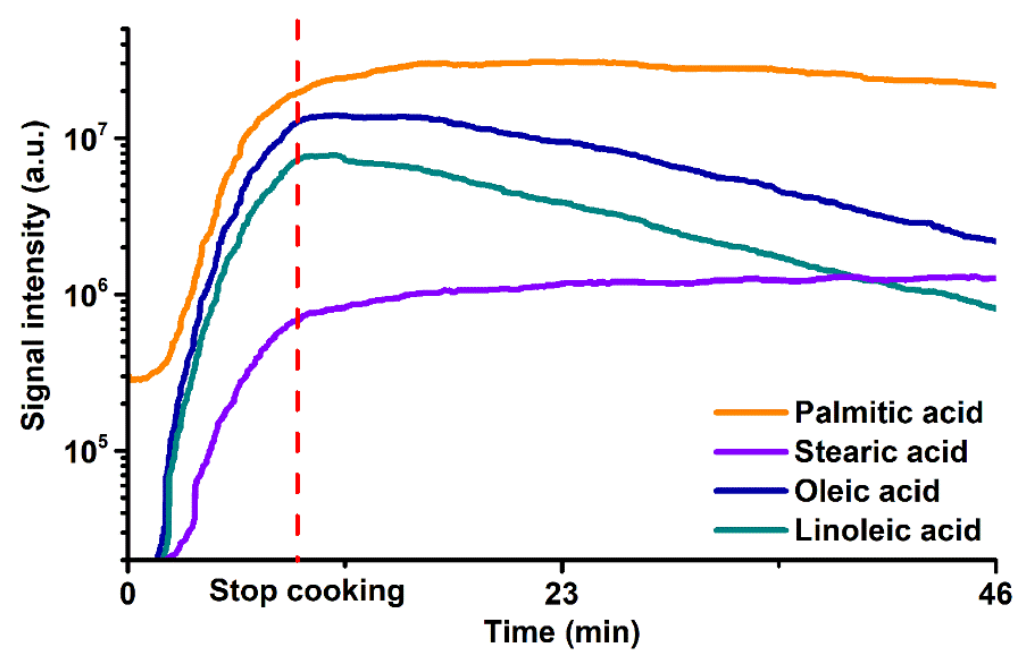




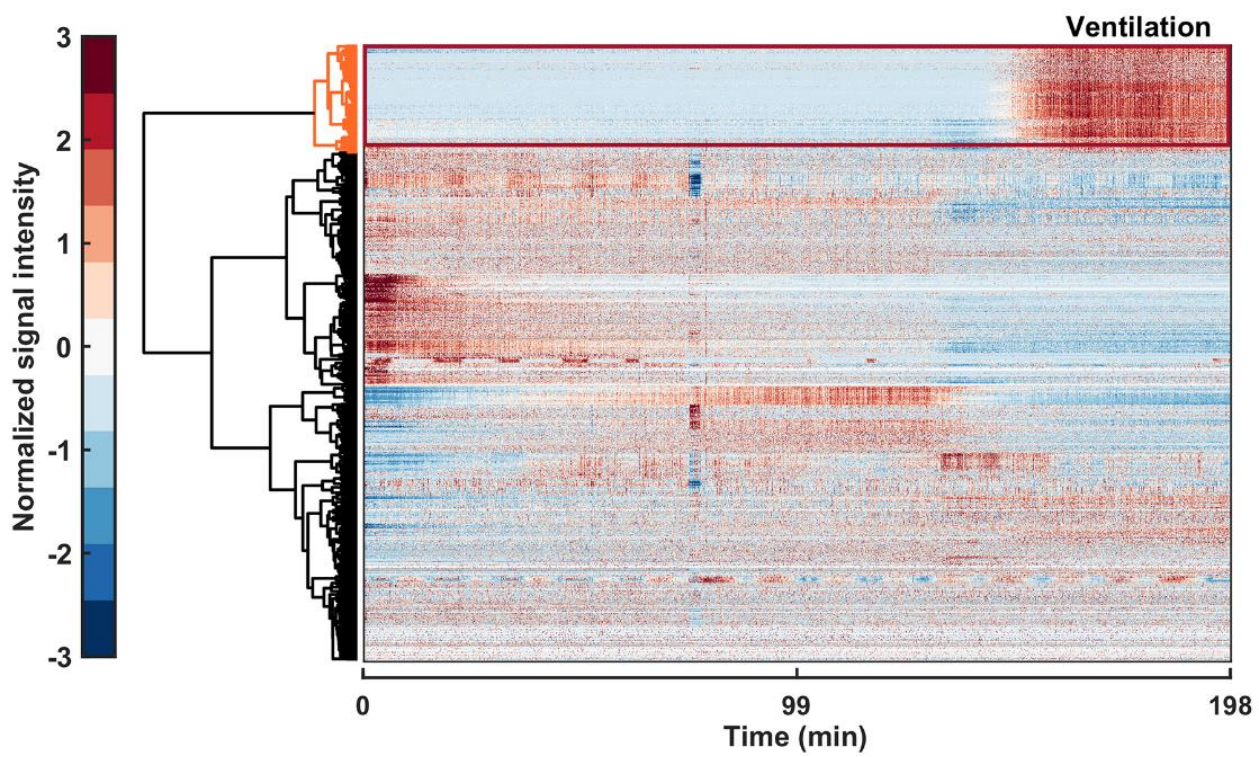

Figure S5. Cluster analysis of ions detected in the control experiment. The features showing up at later stage in top group result from the ventilation.

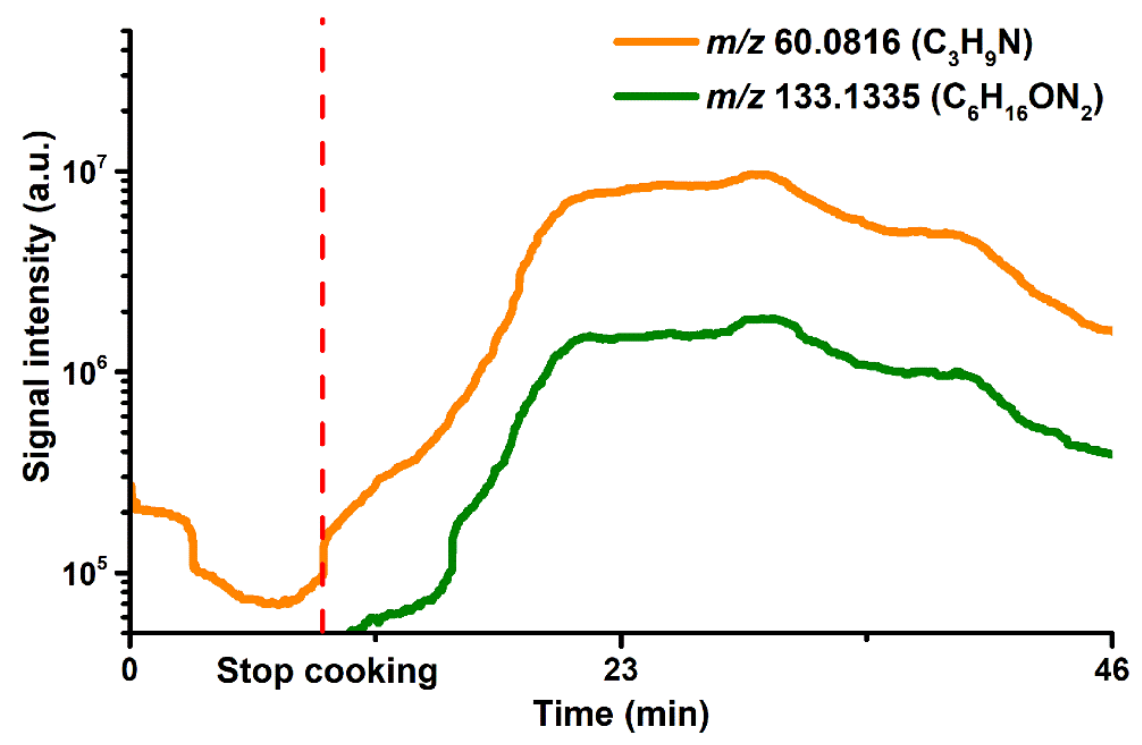

Figure S6. Time-signal intensity profiles of ions detected at $\mathrm{m} / z 60.0816$ and $\mathrm{m} / \mathrm{z}, 133.1335$. 


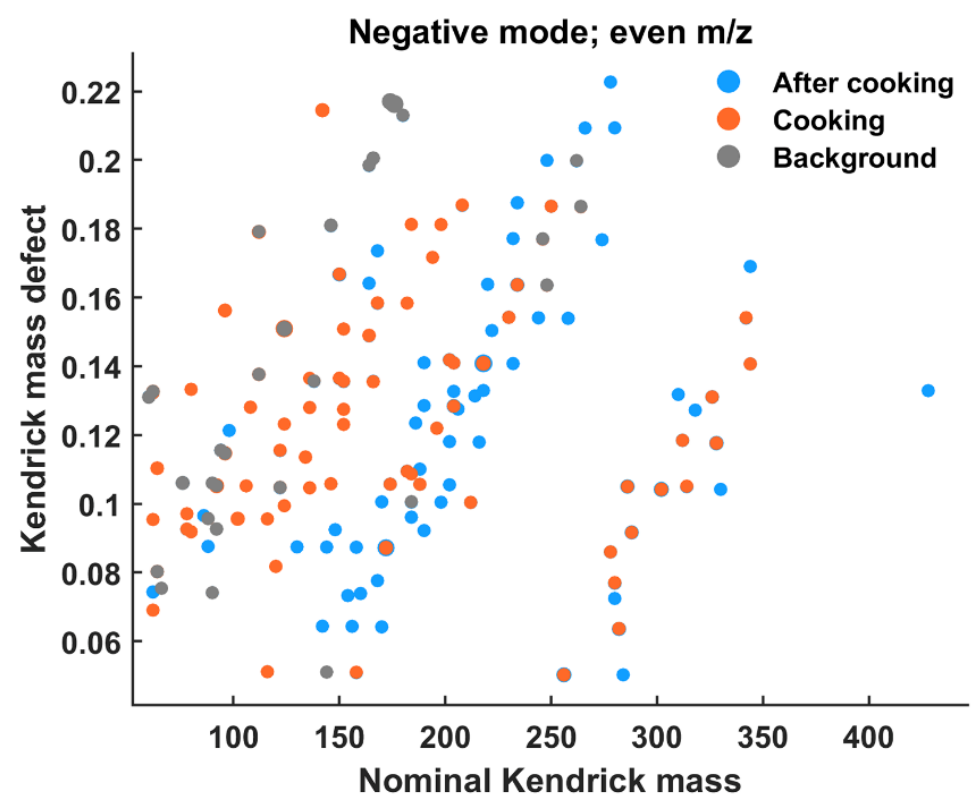

109 Figure S7. KMD plot of even $\mathrm{m} / \mathrm{z}$ values of ions detected in the negative ion detection mode

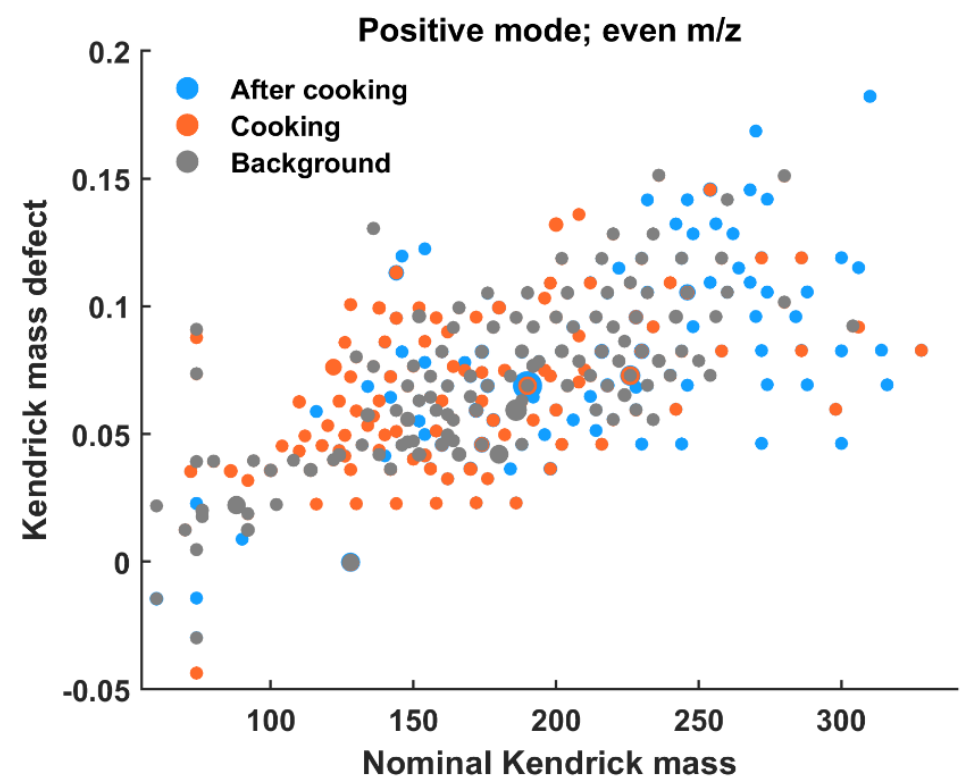

112 Figure S8. KMD plot of even $\mathrm{m} / \mathrm{z}$ values of ions detected in the positive ion detection mode 


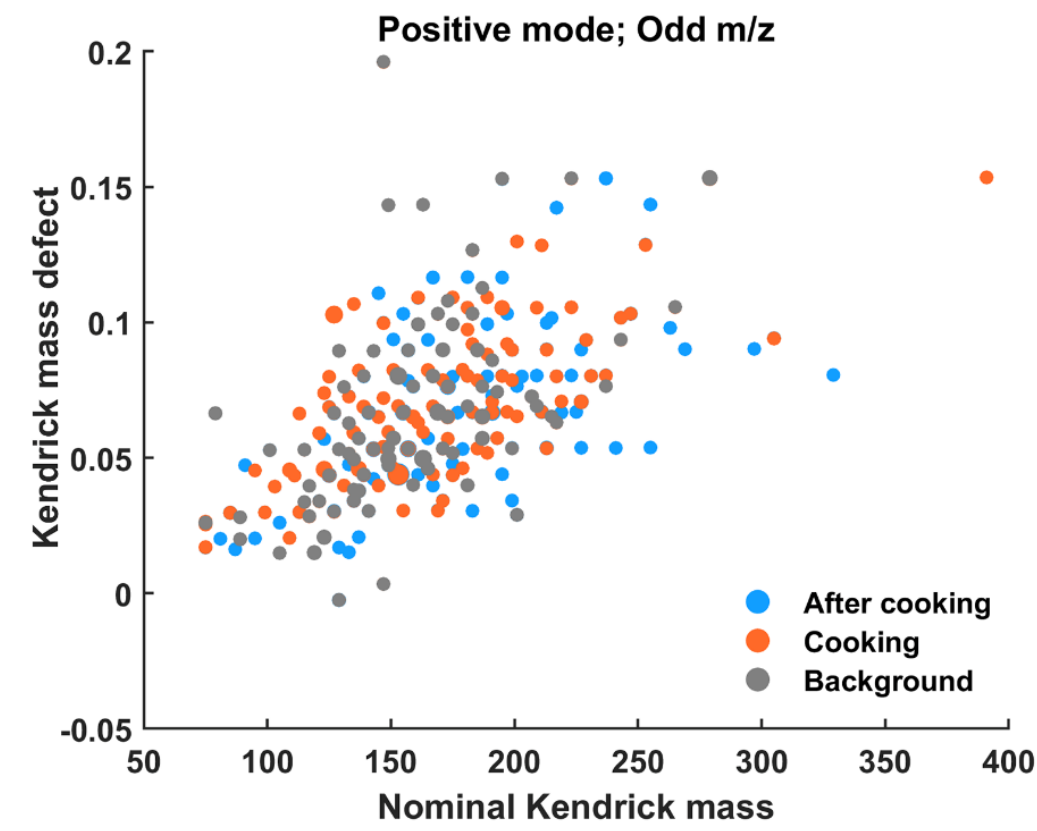

115 Figure S9. KMD plot of odd $\mathrm{m} / \mathrm{z}$ values of ions detected in the positive ion detection mode 


\section{REFERENCES}

118 (1) Madronich, S. Intercomparison of NO2 Photodissociation and U.V. Radiometer Measurements. Atmos.

119 Environ. 1987, 21 (3), 569-578. https://doi.org/10.1016/0004-6981(87)90039-4.

120 (2) Gómez Alvarez, E.; Amedro, D.; Afif, C.; Gligorovski, S.; Schoemaecker, C.; Fittschen, C.; Doussin, J.F.;

121 Wortham, H. Unexpectedly High Indoor Hydroxyl Radical Concentrations Associated with Nitrous Acid. Proc

122 Natl Acad Sci USA 2013, 110 (33), 13294-13299. https://doi.org/10.1073/pnas.1315914110.

123 (3) Liu, J.; Li, S.; Zeng, J.; Mekic, M.; Yu, Z.; Zhou, W.; Loisel, G.; Gandolfo, A.; Song, W.; Wang, X.; Zhou,

124 Z.; Herrmann, H.; Li, X. and Gligorovski, S. Assessing Indoor Gas Phase Oxidation Capacity through Real-

125 Time Measurements of HONO and NO x in Guangzhou, China . Environ. Sci. Process. Impacts 2019, 1393-

126 1402. https://doi.org/10.1039/c9em00194h.

127 (4) Klein, F.; Platt, S. M.; Farren, N. J.; Detournay, A.; Bruns, E. A.; Bozzetti, C.; Daellenbach, K. R.; Kilic,

128 D.; Kumar, N. K.; Pieber, S. M.; et al. Characterization of Gas-Phase Organics Using Proton Transfer Reaction

129 Time-of-Flight Mass Spectrometry: Cooking Emissions. Environ. Sci. Technol. 2016, 50, 1243-1250.

130 https://doi.org/10.1021/acs.est.5b04618.

131 (5) Liu, T.; Wang, Z.; Huang, D. D.; Wang, X.; Chan, C. K. Significant Production of Secondary Organic 132 Aerosol from Emissions of Heated Cooking Oils. Environ. Sci. Technol. Lett. 2018, 5, 32-37.

133 https://doi.org/10.1021/acs.estlett.7b00530.

134 (6) Schauer, J. J.; Kleeman, M. J.; Cass, G.R. and Simoneit, B. R. T. Measurement of Emissions from Air 135 Pollution Sources. 4. C1-C27 Organic Compounds from Cooking with Seed Oils. Environ. Sci. Technol. 2002, 36, 567-575. https://doi.org/10.1021/es002053m.

137 (7) Liu, T.; Liu, Q.; Li, Z.; Huo, L.; Chan, M. N.; Li, X.; Zhou, Z.; Chan, C. K. Emission of Volatile Organic 138 Compounds and Production of Secondary Organic Aerosol from Stir-Frying Spices. Sci. Total Environ. 2017, 139 599-600, 1614-1621. https://doi.org/10.1016/j.scitotenv.2017.05.147.

140 (8) Liu, X. J.; Jin, Q. Z.; Liu, Y. F.; Huang, J. H.; Wang, X. G.; Mao, W. Y.; Wang, S. S. Changes in Volatile 141 Compounds of Peanut Oil during the Roasting Process for Production of Aromatic Roasted Peanut Oil. J. Food 142 Sci. 2011, 76 (3), 404-412. https://doi.org/10.1111/j.1750-3841.2011.02073.x.

143 (9) Hu, W.; Zhang, L.; Li, P.; Wang, X.; Zhang, Q. Characterization of Volatile Components in Four 144 Vegetable Oils by Headspace Two-Dimensional Comprehensive Chromatography Time-of- Fl Ight Mass 145 Spectrometry. Talanta 2014, 129, 629-635. https://doi.org/10.1016/j.talanta.2014.06.010. 
146 (10) Zhao, Y.; Hu, M. I. N. Chemical Compositions of Fine Particulate Organic Matter Emitted from Chinese 147 Cooking. Environ. Sci. Technol. 2007, 41, 99-105. https://doi.org/10.1021/es0614518.

148 (11) Vranová, J.; Ciesarová, Z. Furan in Food - a Review. Czech J. Food Sci. 2009, 27 (1), 1-10.

149 (12) He, W.; Nie, L, Tian, G.; Li, J.; Shao, X.; Wang, M.Y.; Study on the Chemical Compositions of VOCs

150 Emitted by Cooking Oils Based on GC-MS. Environ. Sci. 2013, 34 (12), 4605-4611.

151 https://doi.org/10.13227/j.hjkx.2013.12.002.

152 